\title{
Volatility, Valuation Ratios, and Bubbles: An Empirical Measure of Market Sentiment
}

\author{
CAN GAO and IAN W. R. MARTIN
}

\begin{abstract}
We define a sentiment indicator based on option prices, valuation ratios, and interest rates. The indicator can be interpreted as a lower bound on the expected growth in fundamentals that a rational investor would have to perceive to be happy to hold the market. The bound was unusually high in the late 1990s, reflecting dividend growth expectations that in our view were unreasonably optimistic. Our approach exploits two key ingredients. First, we derive a new valuation ratio decomposition that is related to the Campbell-Shiller loglinearization but that resembles the Gordon growth model more closely and has certain other advantages. Second, we introduce a volatility index that provides a lower bound on the market's expected log return.
\end{abstract}

IN THIS PAPER, WE INTRODUCE A MARKET sentiment indicator that exploits two contrasting views of market predictability. One of our aims is to find a useful way of measuring the "bubbliness" of the market. If valuation ratiosthink $P / D$-are high, then one starts to worry. But valuation ratios can be high for good reasons if interest rates are low, or if risk premia are low (and are widely understood to be low), or both.

A vast literature studies the extent to which signals based on valuation ratios are able to forecast market returns and/or measures of dividend growth. Early papers include Keim and Stambaugh (1986), Campbell and Shiller (1988), and Fama and French (1988). More recently, Martin (2017) argues that implied volatility indexes based on option prices can serve as forecasts of

Can Gao is at the Leibniz Institute for Financial Research SAFE, Frankfurt and Imperial College, London. Ian Martin is at London School of Economics. We thank John Campbell, Stefan Nagel, Patrick Bolton, Ander Perez-Orive, Alan Moreira, Paul Schneider, Sarah Mouabbi, Sean Myers, Wei Xiong, the referees, and seminar participants at LSE, Duke University, the NBER Behavioral Finance Workshop, the Federal Reserve Bank of New York, the Sveriges Riksbank, the Bank of England, and the European Central Bank for their comments. Ian Martin is grateful to the ERC for support under Starting Grant 639744. Neither author has any conflicts of interest to disclose.

Correspondence: Ian W. R. Martin, London School of Economics, London, UK. e-mail: iwrmartin@gmail.com.

This is an open access article under the terms of the Creative Commons AttributionNonCommercial-NoDerivs License, which permits use and distribution in any medium, provided the original work is properly cited, the use is non-commercial and no modifications or adaptations are made.

DOI: $10.1111 /$ jofi.13068

(C) 2021 The Authors. The Journal of Finance published by Wiley Periodicals LLC on behalf of American Finance Association 
expected excess returns, and notes that the two classes of predictor variables made opposing forecasts in the late $1990 \mathrm{~s}$, with valuation ratios pointing to low long-run returns and option prices pointing to high short-run returns.

We play the two views of the world off against one other. Consider the classic Gordon growth model, which relates the market's dividend yield to its expected return minus expected dividend growth: $D / P=\mathbb{E}(R-G)$. Roughly speaking, the idea behind the paper is to use option prices to measure $\mathbb{E} R$, and then to calculate the expected growth in fundamentals implicit in market valuationsour sentiment measure-as the difference between the option price index and dividend yield, $\mathbb{E} G=\mathbb{E} R-\mathbb{E}(R-G)$.

Putting this idea into practice is not as easy as it might seem, however. The Gordon growth model relies on the assumptions that expected returns and expected dividend growth are constant over time. The loglinearized identity of Campbell and Shiller (1988) generalizes the Gordon growth model to the empirically relevant case in which these quantities are time-varying. Their identity relates the price-dividend ratio of an asset to its expected future log dividend growth and expected log returns. It is often characterized as indicating that high valuation ratios signal high expected dividend growth or low expected returns (or both).

But expected returns are not the same as expected log returns. We show that high valuations - and low expected log returns-may be consistent with high expected simple returns if log returns are highly volatile, right-skewed, or fat-tailed. Plausibly, all of these conditions were satisfied in the late 1990s. As they are all potential explanations for the rise in valuation ratios at that time, we will need to be careful about the distinction between log returns and simple returns.

Furthermore, we show that while the Campbell-Shiller identity is highly accurate on average, the linearization is most problematic when the pricedividend ratio is far above its long-run mean. At such times-the late 1990s being a leading example-a researcher who uses the Campbell-Shiller loglinearization will conclude that long-run expected returns are even lower, and/or long-run expected dividend growth is even higher, than is actually the case. Thus, the linearization may "cry bubble" too soon.

We propose a new linearization that does not have this feature, but that also relates a measure of dividend yield to expected log returns and dividend growth. Our approach exploits a measure of dividend yield $y_{t}=\log \left(1+D_{t} / P_{t}\right)$ that has the advantage of being in "natural" units, unlike the quantity $d p_{t}=$ $\log D_{t} / P_{t}$ that features in the Campbell-Shiller approach. As a further bonus, the resulting identity bears an even closer resemblance to the traditional Gordon growth model-which it generalizes to allow for time-varying expected returns and dividend growth-than does the Campbell-Shiller loglinearization.

The second ingredient of our paper is a lower bound on expected log returns that plays the role of $\mathbb{E} R$ in the loose description above. The lower bound relies on an assumption on the form of the stochastic discount factor (SDF). This assumption, the modified negative correlation condition, is satisfied, for example, if one takes the perspective of an unconstrained agent who maximizes 
expected utility over next-period wealth, who chooses to invest his or her wealth fully in the market, and whose relative risk aversion is at least one. An attractive feature of this approach is that it allows the investor in question to coexist with other agents who may or may not be rational. Under the modified negative correlation condition, our lower bound on expected log returns can be computed directly from index option prices so is, broadly speaking, a measure of implied volatility.

The paper is organized as follows. Section I outlines our framework. Section II discusses the link between valuation ratios, returns, and dividend growth: it analyzes the properties of the Campbell-Shiller loglinearization, introduces our alternative loglinearization, and studies the predictive relationship between dividend yields and future log returns and log dividend growth. Section III derives the lower bound on expected returns. Section IV combines the preceding sections to introduce the sentiment indicator. Section V documents that our measure is a leading indicator of detrended volume and of a long-term earnings growth forecast index constructed by Nagel and Xu (2019), and explores its relationship with various other measures of financial conditions. Section VI concludes.

\section{Framework}

Our approach has two ingredients. The first is the predictive relationship between valuation ratios, returns, and fundamentals that has been explored in the vast predictability literature starting from Keim and Stambaugh (1986), Campbell and Shiller (1988), and Fama and French (1988), among others. We introduce a novel loglinearization

$$
\mathbb{E}_{t}\left(r_{t+1}-g_{t+1}\right)=\frac{1}{1-\rho} y_{t}-\frac{\rho}{1-\rho} \mathbb{E}_{t} y_{t+1},
$$

where $r_{t+1}$ is the log return on the market, $g_{t+1}$ is log dividend growth, $y_{t}$ is the market dividend yield, and $\rho \approx 0.97$ is a loglinearization constant. If, say, the dividend yield follows an AR(1) process, ${ }^{1}$ then equation (1) implies that

$$
\mathbb{E}_{t}\left(r_{t+1}-g_{t+1}\right)=a_{0}+a_{1} y_{t}
$$

for some constants $a_{0}$ and $a_{1}$. (We derive these and related results in Section II.)

The second ingredient exploits the information in option prices via a strategy introduced by Martin (2017). We assume that the inequality

$$
\operatorname{cov}_{t}\left(M_{t+1} R_{t+1}, \log R_{t+1}\right) \leq 0
$$

holds; as this is closely related to the negative correlation condition (NCC) of Martin (2017), we refer to it as the modified negative correlation condition

\footnotetext{
${ }^{1}$ Our approach could easily accommodate, say, a vector autoregression for $y_{t}$; the key is that one has an empirical procedure that generates a sensible measure of $\mathbb{E}_{t} y_{t+1}$ to be used in (1). Below, we also consider the case in which $y_{t}$ follows an $\operatorname{AR}(2)$ or $\operatorname{AR}(3)$.
} 
$(m N C C)$. Here $M_{t+1}$ denotes an SDF that prices payoffs delivered at time $t+1$ from the perspective of time $t$, and $R_{t+1}$ is the gross return on the market.

If one thinks from the perspective of an investor whose beliefs and risk preferences are consistent with (2) (or with the alternatives mentioned in footnote 1) and (3), then the mNCC holds if this investor-whom we refer to as a representative investor-maximizes utility $\mathbb{E}_{t} u\left(W_{t+1}\right)$, with relative risk aversion $-W u^{\prime \prime}(W) / u^{\prime}(W)$ (which need not be constant) at least one, and chooses to invest his or her wealth fully in the market. This setup allows for the possibility that other investors are irrational and/or face trading constraints; we emphasize, however, that our representative investor is assumed to be unconstrained, and, in particular, to be able to trade in option markets, so that he or she is marginal for option prices. We therefore rule out extreme forms of market segmentation by assumption. (A representative investor in this sense is sufficient, but not necessary, for the mNCC to hold. We discuss more general conditions under which the mNCC holds in Section III.)

In Section III, we show that this representative investor's beliefs must respect the following lower bound on the expected log return on the market, which can be computed directly from option prices:

$$
\mathbb{E}_{t} r_{t+1}-r_{f, t+1} \geq \frac{1}{P_{t}}\left\{\int_{0}^{F_{t}} \frac{\operatorname{put}_{t}(K)}{K} d K+\int_{F_{t}}^{\infty} \frac{\operatorname{call}_{t}(K)}{K} d K\right\}
$$

Starting from the decomposition

$$
\mathbb{E}_{t} g_{t+1}=r_{f, t+1}-\mathbb{E}_{t}\left(r_{t+1}-g_{t+1}\right)+\mathbb{E}_{t}\left(r_{t+1}-r_{f, t+1}\right),
$$

we have a lower bound on the representative investor's expected dividend growth,

$$
\mathbb{E}_{t} g_{t+1} \geq r_{f, t+1}-\left(a_{0}+a_{1} y_{t}\right)+\frac{1}{P_{t}}\left\{\int_{0}^{F_{t}} \frac{\operatorname{put}_{t}(K)}{K} d K+\int_{F_{t}}^{\infty} \frac{\operatorname{call}_{t}(K)}{K} d K\right\},
$$

from (2), (4), and (5).

To implement this inequality, we replace the population coefficients $a_{0}$ and $a_{1}$ by their sample counterparts $\widehat{a}_{0}$ and $\widehat{a}_{1}$, which we estimate by OLS. ${ }^{2}$ We end up with the sentiment indicator

$$
B_{t}=r_{f, t+1}-\left(\widehat{a}_{0}+\widehat{a}_{1} y_{t}\right)+\frac{1}{P_{t}}\left[\int_{0}^{F_{t}} \frac{\operatorname{put}_{t}(K)}{K} d K+\int_{F_{t}}^{\infty} \frac{\operatorname{call}_{t}(K)}{K} d K\right] .
$$

The indicator is high, indicating the possible presence of a bubble, if there is a combination of high interest rates, a high market valuation ratio (i.e., low dividend yield), and high option prices.

\footnotetext{
${ }^{2}$ We discuss the issue of estimation uncertainty in Section IV.B.1.
} 


\section{Fundamentals}

We seek to exploit the information in valuation ratios, following Campbell and Shiller (1988). Denoting by $P_{t+1}, D_{t+1}$, and $R_{t+1}$ the level, dividend, and gross return of the market, respectively, we have

$$
R_{t+1}=\frac{D_{t+1}+P_{t+1}}{P_{t}} .
$$

It follows from (6) that

$$
r_{t+1}-g_{t+1}=p d_{t+1}-p d_{t}+\log \left(1+e^{d p_{t+1}}\right),
$$

where $d p_{t+1}=d_{t+1}-p_{t+1}=\log D_{t+1}-\log P_{t+1}, p d_{t+1}=p_{t+1}-d_{t+1}$, and $g_{t+1}=$ $d_{t+1}-d_{t}$. Campbell and Shiller (1988) linearize the final term in (7) to derive a decomposition of the (log) price-dividend ratio,

$$
p d_{t}=\frac{k}{1-\rho}+\sum_{i=0}^{\infty} \rho^{i} \mathbb{E}_{t}\left(g_{t+1+i}-r_{t+1+i}\right),
$$

where the constants $k$ and $\rho$ are determined by

$$
\rho=\frac{\mu}{1+\mu} \quad \text { and } \quad \frac{k}{1-\rho}=(1+\mu) \log (1+\mu)-\mu \log \mu, \text { where } \mu=e^{\overline{p d}} .
$$

We follow the convention in the literature in writing approximations such as (8) with equals signs. (A number of our results below are in fact exact. We emphasize these as they occur.) We also assume throughout the paper that there are no rational bubbles, as is standard in the literature. Thus, for example, in deriving (8), we are assuming that $\lim _{T \rightarrow \infty} \rho^{T} \mathbb{E}_{t} p d_{T}=0$.

The approximation (8) is often loosely summarized by saying that high valuation ratios signal high expected dividend growth or low expected returns (or both). But expected log returns are not the same as expected returns: ${ }^{3}$ we have

$$
\mathbb{E}_{t} r_{t+1+i}=\log \mathbb{E}_{t} R_{t+1+i}-\frac{1}{2} \operatorname{var}_{t} r_{t+1+i}-\sum_{n=3}^{\infty} \frac{\kappa_{t}^{(n)}\left(r_{t+1+i}\right)}{n !},
$$

where $\kappa_{t}^{(n)}\left(r_{t+1+i}\right)$ is the $n$th conditional cumulant of the log return. (If returns are conditionally lognormal, then the higher cumulants $\kappa_{t}^{(n)}\left(r_{t+1+i}\right)$ are zero for $n \geq 3$.) Thus, high valuations-and low expected log returns-may be consistent with high expected arithmetic returns if log returns are highly volatile, right-skewed, or fat-tailed. Plausibly, all of these conditions were satisfied in the late 1990s. As they are all potential explanations for the rise in valuation

\footnotetext{
${ }^{3}$ And expected log dividend growth is not the same as expected dividend growth. This distinction is less important, however, as the log dividend growth series is less volatile than the log return series.
} 
ratios at that time, ${ }^{4}$ we will need to be careful about the distinction between log returns and simple returns.

Furthermore, the Campbell-Shiller first-order approximation is least accurate when the valuation ratio is far from its mean, as we now show.

RESULT 1 (Campbell-Shiller revisited): The log price-dividend ratio $\mathrm{pd}_{t}$ obeys the following exact decomposition:

$$
p d_{t}=\frac{k}{1-\rho}+\sum_{i=0}^{\infty} \rho^{i}\left(g_{t+1+i}-r_{t+1+i}\right)+\frac{1}{2} \sum_{i=0}^{\infty} \rho^{i} \psi_{t+1+i}\left(1-\psi_{t+1+i}\right)\left(p d_{t+1+i}-\overline{p d}\right)^{2},
$$

where the constants $k$ and $\rho$ are defined as above, and the quantities $\psi_{t+1+i}$ lie between $\rho$ and $1 /\left(1+e^{d p_{t+1+i}}\right)$.

Equation (9) becomes a second-order Taylor approximation if $\psi_{t}$ is assumed equal to $\rho$ for all $t$,

$$
p d_{t}=\frac{k}{1-\rho}+\sum_{i=0}^{\infty} \rho^{i}\left(g_{t+1+i}-r_{t+1+i}\right)+\frac{\rho(1-\rho)}{2} \sum_{i=0}^{\infty} \rho^{i}\left(p d_{t+1+i}-\overline{p d}\right)^{2},
$$

and reduces to the Campbell-Shiller loglinearization (8) if the final term on the right-hand side of (9) is neglected entirely.

Proof: Taylor's theorem, with the Lagrange form of the remainder, states that for any sufficiently well-behaved function $f$, and for $x \in \mathbb{R}$ and $a \in \mathbb{R}$,

$$
f(x)=f(a)+(x-a) f^{\prime}(a)+\frac{1}{2}(x-a)^{2} f^{\prime \prime}(\xi), \text { for some } \xi \text { between } a \text { and } x .
$$

We apply this result with $f(x)=\log \left(1+e^{x}\right), x=d p_{t+1}$, and $a=\overline{d p}=\mathbb{E} d p_{t}$ equal to the mean log dividend yield. Equation (11) becomes

$$
\log \left(1+e^{d p_{t+1}}\right)=k+(1-\rho) d p_{t+1}+\frac{1}{2} \psi_{t+1}\left(1-\psi_{t+1}\right)\left(d p_{t+1}-\overline{d p}\right)^{2},
$$

where $\psi_{t+1}=1 /\left(1+e^{\xi}\right)$ must lie between $1 /\left(1+e^{\overline{d p}}\right)=\rho$ and $1 /\left(1+e^{d p_{t+1}}\right)$.

Substituting into expression (7), we have the exact relationship

$$
r_{t+1}-g_{t+1}=k-p d_{t}+\rho p d_{t+1}+\frac{1}{2} \psi_{t+1}\left(1-\psi_{t+1}\right)\left(p d_{t+1}-\overline{p d}\right)^{2},
$$

which can be solved forward to give (9). The approximation (10) follows.

Result 1 expresses the price-dividend ratio in terms of future log dividend growth and future log returns-as in the Campbell-Shiller approximationplus a convexity correction.

\footnotetext{
${ }^{4}$ See, for example, Pastor and Veronesi (2003, 2006).
} 
This convexity correction is small on average. Take the unconditional expectation of second-order approximation (10),

$$
\mathbb{E} p d_{t}=\frac{k}{1-\rho}+\frac{\mathbb{E}\left(g_{t}-r_{t}\right)}{1-\rho}+\frac{\rho}{2} \operatorname{var} p d_{t},
$$

assuming that $p d_{t}, r_{t}$, and $g_{t}$ are stationary so that their unconditional means and variances are well defined. Using CRSP data from 1947 to 2019, the sample average of $p d_{t}$ is 3.469 (so that $\rho$ is 0.969 ) and the sample standard deviation is 0.434 . Thus the unconditional average convexity correction $\frac{\rho}{2} \operatorname{var} p d_{t}$ is about 0.0913 , that is, about $2.63 \%$ of the size of $\mathbb{E} p d_{t}$.

The convexity correction can sometimes be large, however. We have

$$
p d_{t}=\frac{k}{1-\rho}+\sum_{i=0}^{\infty} \rho^{i} \mathbb{E}_{t}\left(g_{t+1+i}-r_{t+1+i}\right)+\frac{\rho(1-\rho)}{2} \sum_{i=0}^{\infty} \rho^{i} \mathbb{E}_{t}\left(p d_{t+1+i}-\overline{p d}\right)^{2},
$$

and the final term may be quantitatively important if the valuation ratio is far from its mean and persistent, so that it is expected to remain far from its mean for a significant length of time.

For the sake of argument, suppose that the log price-dividend ratio follows an $\operatorname{AR}(1), p d_{t+1}-\overline{p d}=\phi\left(p d_{t}-\overline{p d}\right)+\varepsilon_{t+1}$, where $\operatorname{var}_{t} \varepsilon_{t+1}=\sigma^{2}$ so that $\operatorname{var} p d_{t}=\sigma^{2} /\left(1-\phi^{2}\right)$, and set $\sigma=0.167$ and $\phi=0.923$ to match the sample standard deviation and autocorrelation in CRSP data from 1947 to 2019. The above expression becomes

$$
p d_{t}=\frac{k}{1-\rho}+\sum_{i=0}^{\infty} \rho^{i} \mathbb{E}_{t}\left(g_{t+1+i}-r_{t+1+i}\right)+\underbrace{\frac{\rho(1-\rho) \phi^{2}}{2\left(1-\rho \phi^{2}\right)}\left[\left(p d_{t}-\overline{p d}\right)^{2}+\frac{\sigma^{2}}{(1-\rho) \phi^{2}}\right]}_{\text {convexity correction }} .
$$

At its peak during the boom of the late $1990 \mathrm{~s}, p d_{t}$ was 2.2 standard deviations above its mean. The convexity term then equals 0.145 : this is the amount by which a researcher using the Campbell-Shiller approximation would overstate $\sum_{i=0}^{\infty} \rho^{i} \mathbb{E}_{t}\left(g_{t+1+i}-r_{t+1+i}\right)$. With $\rho=0.969$, this is equivalent to overstating $\mathbb{E}_{t} g_{t+1+i}-r_{t+1+i}$ by 14.5 percentage points for one year, 3.1 percentage points for five years, or 1.0 percentage points for 20 years. ${ }^{5}$

The Campbell-Shiller approximation does not apply if $d p_{t}$ follows a random walk (i.e., $\mathbb{E}_{t} d p_{t+1}=d p_{t}$ ). But in that case, we can linearize (7) around the

\footnotetext{
5 The numbers are more dramatic if we use the long sample from 1871 to 2015 available on Robert Shiller's website. We find $\rho=0.960, \sigma=0.136$, and $\phi=0.942$ in the long sample, so that the convexity correction is 0.0596 when $p d_{t}$ is at its mean and 0.253 at the peak (which is 3.2 standard deviations above the mean). This last number corresponds to overstating $\mathbb{E}_{t} g_{t+1+i}-$ $r_{t+1+i}$ by 25.3 percentage points for one year, 5.5 percentage points for five years, 1.8 percentage points for 20 years, or 1.0 percentage points forever.
} 
conditional mean $\mathbb{E}_{t} d p_{t+1}$ to find ${ }^{6}$

$$
\mathbb{E}_{t}\left(r_{t+1}-g_{t+1}\right)=\log \left(1+e^{d p_{t}}\right)=\log \left(1+\frac{D_{t}}{P_{t}}\right) .
$$

Motivated by this fact, ${ }^{7}$ we define $y_{t}=\log \left(1+D_{t} / P_{t}\right)$. An appealing property of this definition-and one that $d p_{t}$ does not possess-is that $y_{t}=\log (1+$ $\left.D_{t} / P_{t}\right) \approx D_{t} / P_{t}$. We can then rewrite the definition of the log return (7) as the (exact) relationship

$$
r_{t+1}-g_{t+1}=y_{t}+\log \left(1-e^{-y_{t}}\right)-\log \left(1-e^{-y_{t+1}}\right) .
$$

In these terms, equation (12) states that

$$
y_{t}=\mathbb{E}_{t}\left(r_{t+1}-g_{t+1}\right),
$$

which is valid, as a first-order approximation, if $d p_{t}$ (or $y_{t}$ ) follows a random walk.

Alternatively, if $y_{t}$ is stationary (as is almost always assumed in the literature), we have the following result. We write unconditional means as $\bar{y}=\mathbb{E} y_{t}$, $\bar{r}=\mathbb{E} r_{t}$, and $\bar{g}=\mathbb{E} g_{t}$.

RESULT 2 (The Gordon growth model, generalized): Suppose that $y_{t}$ is stationary. Then we have the loglinearization

$$
y_{t}=(1-\rho) \sum_{i=0}^{\infty} \rho^{i}\left(r_{t+1+i}-g_{t+1+i}\right),
$$

where ${ }^{8} \rho=e^{-\bar{y}}$. As there is no constant in (15), and as $(1-\rho) \sum_{i=0}^{\infty} \rho^{i}=1$, this is a variant of the Gordon growth model: $y$ is a weighted average of future $r-g$.

To second order, we have the approximation

$$
y_{t}=(1-\rho) \sum_{i=0}^{\infty} \rho^{i}\left(r_{t+1+i}-g_{t+1+i}\right)-\frac{1}{2} \frac{\rho}{1-\rho} \sum_{i=0}^{\infty} \rho^{i}\left[\left(y_{t+1+i}-\bar{y}\right)^{2}-\left(y_{t+i}-\bar{y}\right)^{2}\right] \text {. }
$$

We also have the exact relationship

$$
\bar{y}=\bar{r}-\bar{g},
$$

which does not rely on any approximation.

\footnotetext{
${ }^{6}$ Campbell $(2008,2018)$ derives the same result via a different route, but makes further assumptions (namely, that the driving shocks are homoskedastic and conditionally Normal) that we do not require.

${ }^{7}$ Further motivation is provided by Martin (2013), who shows that this measure of dividend yield emerges naturally in i.i.d. models with power utility or Epstein-Zin (1989) preferences.

8 This differs slightly from the definition of $\rho$ in Result 1 , although they are extremely close in practice.
} 
Proof: Using Taylor's theorem to second order in equation (13), we have the second-order approximation

$$
r_{t+1}-g_{t+1}=\frac{1}{1-\rho} y_{t}-\frac{\rho}{1-\rho} y_{t+1}+\frac{1}{2} \frac{\rho}{(1-\rho)^{2}}\left[\left(y_{t+1}-\bar{y}\right)^{2}-\left(y_{t}-\bar{y}\right)^{2}\right],
$$

which can be rewritten

$$
y_{t}=(1-\rho)\left(r_{t+1}-g_{t+1}\right)+\rho y_{t+1}-\frac{1}{2} \frac{\rho}{1-\rho}\left[\left(y_{t+1}-\bar{y}\right)^{2}-\left(y_{t}-\bar{y}\right)^{2}\right]
$$

and then solved forward, giving (15) and (16). Equation (17) follows by taking expectations of the identity (13) and noting that $\mathbb{E} \log \left(1-e^{-y_{t}}\right)=\mathbb{E} \log (1-$ $\left.e^{-y_{t+1}}\right)$ by stationarity of $y_{t}$.

We note in passing that equation (17) implies that the inequality $\bar{r}>\bar{g}$, which is discussed extensively by Piketty (2014), holds in any model in which $\bar{y}>0$. Piketty (2015) writes that "the inequality $r>g$ holds true in the steady-state equilibrium of the most common economic models, including representativeagent models where each individual owns an equal share of the capital stock." Our result shows that the inequality applies much more generally. It does not rely on equilibrium logic and is not in itself particularly interesting or significant.

Given our focus on bubbles, we are particularly interested in the accuracy of these loglinearizations ${ }^{9}$ at times when valuation ratios are unusually high or, equivalently, when $d p_{t}$ and $y_{t}$ are unusually low. This motivates the following definition and result.

DEFINITION 1: We say that $y_{t}$ is far from its mean (at time $t$ ) if

$$
\mathbb{E}_{t}\left[\left(y_{t+1+i}-\bar{y}\right)^{2}\right] \leq\left(y_{t}-\bar{y}\right)^{2} \quad \text { for all } i \geq 0 .
$$

Example: If $y_{t}$ follows an AR(1), then a direct calculation shows that $y_{t}$ is far from its mean if and only if it is at least one standard deviation from its mean.

RESULT 3 (Signing the approximation errors): We can sign the approximation error in the Campbell-Shiller loglinearization (8):

$$
d p_{t}<-\frac{k}{1-\rho}+\sum_{i=0}^{\infty} \rho^{i} \mathbb{E}_{t}\left(r_{t+1+i}-g_{t+1+i}\right) .
$$

\footnotetext{
${ }^{9}$ In a different direction, motivation for doing so is provided by Pohl, Schmedders, and Wilms (2018), who show that loglinearizations can induce quantitatively important approximation errors in long-run risk models.
} 
The first-order approximation (15) is exact on average, that is,

$$
\mathbb{E} y_{t}=(1-\rho) \sum_{i=0}^{\infty} \rho^{i} \mathbb{E}\left(r_{t+1+i}-g_{t+1+i}\right)
$$

holds exactly, without any approximation. But if $y_{t}$ is far from its mean, then (up to a second-order approximation)

$$
y_{t} \geq(1-\rho) \sum_{i=0}^{\infty} \rho^{i} \mathbb{E}_{t}\left(r_{t+1+i}-g_{t+1+i}\right) .
$$

Proof: The inequality (19) follows immediately from (9), and equation (20) follows directly from equation (17). To establish the inequality (21), rewrite

$$
\begin{aligned}
\sum_{i=0}^{\infty} \rho^{i}\left[\left(y_{t+1+i}-\bar{y}\right)^{2}-\left(y_{t+i}-\bar{y}\right)^{2}\right] & =-\left(y_{t}-\bar{y}\right)^{2}+(1-\rho) \sum_{i=0}^{\infty} \rho^{i}\left(y_{t+1+i}-\bar{y}\right)^{2} \\
& =(1-\rho) \sum_{i=0}^{\infty} \rho^{i}\left[\left(y_{t+1+i}-\bar{y}\right)^{2}-\left(y_{t}-\bar{y}\right)^{2}\right] .
\end{aligned}
$$

The inequality then follows from (16), (18), and (22).

Dividend yields, whether measured by $d p_{t}$ or $y_{t}$, were unusually low around the turn of the millennium, indicating some combination of low future returns and high future dividend growth. Result 3 shows that an econometrician who uses the Campbell-Shiller approximation (8) at such a time-that is, who treats the inequality (19) as an equality-will overstate how low future returns, or how high future dividend growth, must be, and therefore may be too quick to conclude that the market is "bubbly." In contrast, an econometrician who uses the approximation (15) will understate how low future returns, or how high future dividend growth, must be. Thus $y_{t}$ is a conservative diagnostic for bubbles.

To place more structure on the relationship between valuation ratios, $r$, and $g$, we need to make an assumption about the evolution of $d p_{t}$ and $y_{t}$ over time. For now we rely on an AR(1) assumption to keep things simple; in Appendix A, we report corresponding results assuming $\mathrm{AR}(2)$ or $\mathrm{AR}(3)$ processes.

The Campbell-Shiller approximation over one period states that $r_{t+1}-$ $g_{t+1}=k+\underline{d p_{t}}-\rho d p_{t+1}$. If $d p_{t}$ follows an $\operatorname{AR}(1)$ with autocorrelation $\phi$, then $\mathbb{E}_{t} d p_{t+1}-\overline{d p}=\phi\left(d p_{t}-\overline{d p}\right)$, so

$$
\mathbb{E}_{t}\left(r_{t+1}-g_{t+1}\right)=c+(1-\rho \phi) d p_{t},
$$

where we have absorbed constant terms into $c$. 
Table I Full-Sample Regressions for S\&P 500, Annual Data, Cash
Reinvestment, 1947 to 2019

\begin{tabular}{lccccrr}
\hline $\mathrm{RHS}_{t}$ & $\mathrm{LHS}_{t+1}$ & $\widehat{a}_{0}$ & s.e. & $\widehat{a}_{1}$ & s.e. & $R^{2}$ \\
\hline \multirow{4}{*}{$y_{t}$} & $r_{t+1}-g_{t+1}$ & -0.073 & {$[0.048]$} & 3.541 & {$[1.302]$} & $8.33 \%$ \\
& $r_{t+1}$ & -0.023 & {$[0.048]$} & 3.820 & {$[1.194]$} & $11.13 \%$ \\
& $-g_{t+1}$ & -0.050 & {$[0.027]$} & -0.279 & {$[0.802]$} & $0.29 \%$ \\
& $r_{t+1}-g_{t+1}$ & 0.430 & {$[0.144]$} & 0.111 & {$[0.041]$} & $8.23 \%$ \\
& $r_{t+1}$ & 0.511 & {$[0.136]$} & 0.117 & {$[0.040]$} & $10.58 \%$ \\
& $-g_{t+1}$ & -0.081 & {$[0.084]$} & -0.006 & {$[0.024]$} & $0.16 \%$ \\
\hline
\end{tabular}

Conversely, the first-order approximation underlying Result 2 implies that

$$
\mathbb{E}_{t}\left(r_{t+1}-g_{t+1}\right)=\frac{1}{1-\rho} y_{t}-\frac{\rho}{1-\rho} \mathbb{E}_{t} y_{t+1}
$$

If $y_{t}$ follows an $\operatorname{AR}(1)$ with autocorrelation $\phi_{y}$, then this reduces to

$$
\mathbb{E}_{t}\left(r_{t+1}-g_{t+1}\right)=c+\frac{1-\rho \phi_{y}}{1-\rho} y_{t},
$$

where again we absorb constants into the intercept $c$. In view of (17), this can be written without an intercept as

$$
\mathbb{E}_{t}\left(r_{t+1}-g_{t+1}\right)-(\bar{r}-\bar{g})=\frac{1-\rho \phi_{y}}{1-\rho}\left(y_{t}-\bar{y}\right),
$$

so that the deviation of $y_{t}$ from its long-run mean is proportional to the deviation of conditionally expected $r_{t+1}-g_{t+1}$ from its long-run mean. A further advantage of $y_{t}$ over $d p_{t}$ is that the expression (25) is also meaningful if $y_{t}$ follows a random walk: in this case, the coefficient on $y_{t}$ equals one and the intercept is zero, by equation (14).

Equations (23) and (25) motivate regressions of realized $r_{t+1}-g_{t+1}$ onto $d p_{t}$ and a constant, or onto $y_{t}$ and a constant. The results are shown in Table I, where we also report the results of regressing $r_{t+1}$ and $-g_{t+1}$ separately onto $y_{t}$ and $d p_{t}$. We use end-of-year observations of the price level and accumulated dividends of the S\&P 500 index from CRSP. ${ }^{10}$ The table reports regression

\footnotetext{
${ }^{10}$ We calculate the monthly dividend by multiplying the difference between monthly cumdividend and ex-dividend returns by the lagged ex-dividend price: $D_{t}=\left(R_{c u m, t}-R_{\text {ex, } t}\right) P_{t-1}$. As we aggregate the dividends paid out over the year, to address seasonality issues, we reinvest dividends month-by-month until the end of the year, using the CRSP 30-day T-bill rate as our risk-free rate. In Tables III and IV, we report similar results with dividends reinvested at the cum-dividend market return rather than at a risk-free rate; if anything, these results are somewhat more favorable to our $y_{t}$ variable than to $d p_{t}$.
} 
Table II

Predictive Regressions for S\&P 500, Annual Data, Cash Reinvestment, 1926 to 2019

\begin{tabular}{lccccrr}
\hline $\mathrm{RHS}_{t}$ & $\mathrm{LHS}_{t+1}$ & $\widehat{a}_{0}$ & s.e. & $\widehat{a}_{1}$ & s.e. & $R^{2}$ \\
\hline \multirow{4}{*}{$y_{t}$} & $r_{t+1}-g_{t+1}$ & -0.145 & {$[0.053]$} & 5.120 & {$[1.492]$} & $13.40 \%$ \\
& $r_{t+1}$ & 0.028 & {$[0.051]$} & 1.775 & {$[1.361]$} & $2.26 \%$ \\
& $-g_{t+1}$ & -0.173 & {$[0.050]$} & 3.345 & {$[1.490]$} & $16.29 \%$ \\
\multirow{2}{*}{$d p_{t}$} & $r_{t+1}-g_{t+1}$ & 0.557 & {$[0.185]$} & 0.152 & {$[0.052]$} & $9.54 \%$ \\
& $r_{t+1}$ & 0.330 & {$[0.154]$} & 0.070 & {$[0.045]$} & $2.86 \%$ \\
& $-g_{t+1}$ & 0.227 & {$[0.161]$} & 0.082 & {$[0.046]$} & $7.85 \%$ \\
\hline
\end{tabular}

Table III

Predictive Regressions for S\&P 500, Annual Data, Market Reinvestment, 1947 to 2019

\begin{tabular}{lccccrr}
\hline $\mathrm{RHS}_{t}$ & $\mathrm{LHS}_{t+1}$ & $\widehat{a}_{0}$ & s.e. & $\widehat{a}_{1}$ & s.e. & \multicolumn{1}{c}{$R^{2}$} \\
\hline \multirow{4}{*}{$y_{t}$} & $r_{t+1}-g_{t+1}$ & -0.031 & {$[0.040]$} & 2.290 & {$[1.073]$} & $6.91 \%$ \\
& $r_{t+1}$ & -0.018 & {$[0.049]$} & 3.554 & {$[1.154]$} & $10.43 \%$ \\
& $-g_{t+1}$ & -0.013 & {$[0.045]$} & -1.264 & {$[1.228]$} & $2.30 \%$ \\
\multirow{2}{*}{$d p_{t}$} & $r_{t+1}-g_{t+1}$ & 0.311 & {$[0.123]$} & 0.076 & {$[0.036]$} & $6.92 \%$ \\
& $r_{t+1}$ & 0.519 & {$[0.137]$} & 0.120 & {$[0.041]$} & $10.76 \%$ \\
& $-g_{t+1}$ & -0.208 & {$[0.135]$} & -0.044 & {$[0.039]$} & $2.51 \%$ \\
\hline
\end{tabular}

results of the form ${ }^{11}$

$$
\mathrm{LHS}_{t+1}=a_{0}+a_{1} \times \mathrm{RHS}_{t}+\varepsilon_{t+1},
$$

with Hansen-Hodrick (1980) standard errors shown in brackets. (Under the $\operatorname{AR}(1)$ assumption, we could also use (23) or (25) as estimates of $\mathbb{E}_{t}\left(r_{t+1}-\right.$ $\left.g_{t+1}\right)$. This approach yields very similar results, as we show in Table A.I of Appendix A.)

The variables $y_{t}$ and $d p_{t}$ have similar predictive performance and, consistent with prior literature, we find that valuation ratios help to forecast returns in the post-1947 sample but have limited forecasting power for dividend growth. We have also considered variations on this exercise. Table II reports results using cash-reinvested dividends in the full CRSP post-1926 sample. Tables III and IV report results using market-reinvested dividends in the post-1947 and the post-1926 period, respectively. Tables V to VII use the price and dividend data of Goyal and Welch (2008) (updated to 2018 and taken from Amit Goyal's webpage); this gives us a longer sample, as it incorporates Robert Shiller's

\footnotetext{
${ }^{11}$ Stambaugh bias has little effect on these numbers. In our main sample, implementing the correction given in equation (18) of Stambaugh (1999) changes the predictive coefficient from 3.54 to 3.35 in the data since 1947 , or from 5.12 to 5.11 in the data since 1926 . We therefore report simple OLS coefficients for simplicity. The analysis of Section IV.B.2 can also be interpreted as a conservative response to the possibility of Stambaugh bias.
} 
Table IV

Predictive Regressions for S\&P 500, Annual Data, Market

Reinvestment, 1926 to 2019

\begin{tabular}{lcccccc}
\hline $\mathrm{RHS}_{t}$ & $\mathrm{LHS}_{t+1}$ & $\widehat{a}_{0}$ & s.e. & $\widehat{a}_{1}$ & s.e. & $R^{2}$ \\
\hline \multirow{4}{*}{$y_{t}$} & $r_{t+1}-g_{t+1}$ & -0.063 & {$[0.041]$} & 2.921 & {$[1.123]$} & $8.38 \%$ \\
& $r_{t+1}$ & -0.015 & {$[0.048]$} & 2.875 & {$[1.138]$} & $5.16 \%$ \\
& $-g_{t+1}$ & -0.048 & {$[0.040]$} & 0.046 & {$[1.003]$} & $0.00 \%$ \\
\multirow{2}{*}{$d p_{t}$} & $r_{t+1}-g_{t+1}$ & 0.347 & {$[0.130]$} & 0.089 & {$[0.038]$} & $6.52 \%$ \\
& $r_{t+1}$ & 0.411 & {$[0.142]$} & 0.095 & {$[0.042]$} & $4.66 \%$ \\
& $-g_{t+1}$ & -0.064 & {$[0.120]$} & -0.005 & {$[0.035]$} & $0.03 \%$ \\
\hline
\end{tabular}

Table V

Predictive Regressions for S\&P500, Annual Data from Amit Goyal's Website, 1947 to 2018

\begin{tabular}{lccccrr}
\hline $\mathrm{RHS}_{t}$ & $\mathrm{LHS}_{t+1}$ & $\widehat{a}_{0}$ & s.e. & $\widehat{a}_{1}$ & s.e. & $R^{2}$ \\
\hline \multirow{3}{*}{$y_{t}$} & $r_{t+1}-g_{t+1}$ & -0.070 & {$[0.048]$} & 3.549 & {$[1.289]$} & $8.26 \%$ \\
& $r_{t+1}$ & -0.020 & {$[0.047]$} & 3.807 & {$[1.182]$} & $10.84 \%$ \\
& $-g_{t+1}$ & -0.050 & {$[0.023]$} & -0.257 & {$[0.734]$} & $0.31 \%$ \\
& $r_{t+1}-g_{t+1}$ & 0.435 & {$[0.141]$} & 0.111 & {$[0.040]$} & $8.46 \%$ \\
& $r_{t+1}$ & 0.511 & {$[0.134]$} & 0.116 & {$[0.039]$} & $10.54 \%$ \\
& $-g_{t+1}$ & -0.076 & {$[0.072]$} & -0.005 & {$[0.020]$} & $0.12 \%$ \\
\hline
\end{tabular}

Table VI

Predictive Regressions for S\&P500, Annual Data from Amit Goyal's Website, 1926 to 2018

\begin{tabular}{lcrcrrr}
\hline $\mathrm{RHS}_{t}$ & $\mathrm{LHS}_{t+1}$ & \multicolumn{1}{c}{$\widehat{a}_{0}$} & s.e. & $\widehat{a}_{1}$ & s.e. & $R^{2}$ \\
\hline \multirow{3}{*}{$y_{t}$} & $r_{t+1}-g_{t+1}$ & -0.120 & {$[0.049]$} & 4.470 & {$[1.369]$} & $12.05 \%$ \\
& $r_{t+1}$ & 0.033 & {$[0.049]$} & 1.628 & {$[1.343]$} & $2.04 \%$ \\
& $-g_{t+1}$ & -0.153 & {$[0.040]$} & 2.842 & {$[1.213]$} & $17.07 \%$ \\
\multirow{2}{*}{$d p_{t}$} & $r_{t+1}-g_{t+1}$ & 0.500 & {$[0.169]$} & 0.135 & {$[0.048]$} & $8.84 \%$ \\
& $r_{t+1}$ & 0.315 & {$[0.153]$} & 0.065 & {$[0.044]$} & $2.67 \%$ \\
& $-g_{t+1}$ & 0.186 & {$[0.133]$} & 0.069 & {$[0.037]$} & $8.17 \%$ \\
\hline
\end{tabular}

Table VII

Predictive Regressions for S\&P500, Annual Data from Amit Goyal's Website, 1871 to 2018

\begin{tabular}{lccccrr}
\hline $\mathrm{RHS}_{t}$ & $\mathrm{LHS}_{t+1}$ & $\widehat{a}_{0}$ & s.e. & $\widehat{a}_{1}$ & s.e. & $R^{2}$ \\
\hline \multirow{3}{*}{$y_{t}$} & $r_{t+1}-g_{t+1}$ & -0.143 & {$[0.041]$} & 4.500 & {$[0.968]$} & $13.03 \%$ \\
& $r_{t+1}$ & 0.043 & {$[0.038]$} & 0.992 & {$[0.872]$} & $0.89 \%$ \\
& $-g_{t+1}$ & -0.185 & {$[0.030]$} & 3.507 & {$[0.769]$} & $22.91 \%$ \\
& $r_{t+1}-g_{t+1}$ & 0.501 & {$[0.125]$} & 0.140 & {$[0.037]$} & $9.00 \%$ \\
& $r_{t+1}$ & 0.217 & {$[0.108]$} & 0.041 & {$[0.033]$} & $1.07 \%$ \\
& $-g_{t+1}$ & 0.284 & {$[0.093]$} & 0.099 & {$[0.028]$} & $13.09 \%$ \\
\hline
\end{tabular}


data, which go back as far as 1871 . The predictability of $r$ relative to $g$ is to some extent a feature of the postwar period. In the long sample, returns are substantially less predictable and dividends substantially more predictable, perhaps because of the postwar tendency of corporations to smooth dividends (Lintner (1956)). Encouragingly, however, we find that the predictive relationship between $y_{t}$ (or $d p_{t}$ ) and the difference $r_{t+1}-g_{t+1}$ is fairly stable across sample periods and data sources.

\section{A Lower Bound on Expected Log Returns}

High valuation ratios are sometimes cited as direct evidence of a bubble. But valuation ratios can be high for good reasons if interest rates or rationally expected risk premia are low. In other words, if we use $y_{t}$ to measure $\mathbb{E}_{t}\left(r_{t+1}-\right.$ $\left.g_{t+1}\right)$ as suggested above, we may find that $y_{t}$ is low simply because $\mathbb{E}_{t} r_{t+1}$ is very low, which could reflect low interest rates $r_{f, t+1}$, low (log) risk premia $\mathbb{E}_{t} r_{t+1}-r_{f, t+1}$, or both.

While interest rates are directly observable, risk premia are harder to measure. We start from the following identity, which generalizes an identity introduced by Martin (2017) in the case $X_{t+1}=R_{t+1}$ :

$$
\mathbb{E}_{t} X_{t+1}=\frac{1}{R_{f, t+1}} \mathbb{E}_{t}^{*}\left(R_{t+1} X_{t+1}\right)-\operatorname{cov}_{t}\left(M_{t+1} R_{t+1}, X_{t+1}\right)
$$

We write $\mathbb{E}_{t}^{*}$ for the time $t$ conditional risk-neutral expectation operator, defined by the property that $\frac{1}{R_{f, t+1}} \mathbb{E}_{t}^{*} X_{t+1}=\mathbb{E}_{t}\left(M_{t+1} X_{t+1}\right)$, where $M_{t+1}$ denotes an SDF that prices any tradable payoff $X_{t+1}$ received at time $t+1$. Assuming the absence of arbitrage, such an SDF must exist, and the identity above holds for any gross return $R_{t+1}$ such that the payoff $R_{t+1} X_{t+1}$ is tradable. Henceforth, however, $R_{t+1}$ will always denote the gross return on the market.

We are interested in expected $\log$ returns, $X_{t+1}=\log R_{t+1}$, in which case the identity becomes

$$
\mathbb{E}_{t} \log R_{t+1}=\frac{1}{R_{f, t+1}} \mathbb{E}_{t}^{*}\left(R_{t+1} \log R_{t+1}\right)-\operatorname{cov}_{t}\left(M_{t+1} R_{t+1}, \log R_{t+1}\right)
$$

The first of the two terms on the right-hand side, as a risk-neutral expectation, is directly observable from asset prices, as it represents the price of a contract that pays $R_{t+1} \log R_{t+1}$ at time $t+1$. (Neuberger (2012) studies this contract in a different context.) The second term can be controlled: we argue below that it is reasonable to impose the assumption that it is negative. The identity (26) then implies a lower bound on expected log returns in terms of a quantity that is directly observable from asset prices.

To make further progress, we make two assumptions throughout the paper. As we show below, we can use option prices to bound the first term on the 
right-hand side of the identity (26). Our first assumption addresses the minor ${ }^{12}$ technical issue that we observe options on the ex-dividend value of the index, $P_{t+1}$, rather than on $P_{t+1}+D_{t+1}$.

Assumption 1: If we define the dispersion measure $\Psi\left(X_{t+1}\right) \equiv \mathbb{E}_{t}^{*} f\left(X_{t+1}\right)-$ $f\left(\mathbb{E}_{t}^{*} X_{t+1}\right)$, where $f(x)=x \log x$ is a convex function, then the dispersion of $R_{t+1}$ is at least as large as that of $P_{t+1} / P_{t}$ :

$$
\Psi\left(R_{t+1}\right) \geq \Psi\left(P_{t+1} / P_{t}\right)
$$

This condition is very mild. Expanding $f(x)=x \log x$ as a Taylor series to second order around $x=1, f(x) \approx\left(x^{2}-1\right) / 2$. Thus, to second order, Assumption 1 is equivalent to $\operatorname{var}_{t}^{*} R_{t+1} \geq \operatorname{var}_{t}^{*}\left(P_{t+1} / P_{t}\right)$, or to $\operatorname{var}_{t}^{*}\left(P_{t+1}+D_{t+1}\right) \geq \operatorname{var}_{t}^{*} P_{t+1}$. A sufficient, though not necessary, condition for this to hold is that the price $P_{t+1}$ and dividend $D_{t+1}$ are weakly positively correlated under the risk-neutral measure.

Our second assumption is more substantive.

Assumption 2: The mNCC holds:

$$
\operatorname{cov}_{t}\left(M_{t+1} R_{t+1}, \log R_{t+1}\right) \leq 0 .
$$

Martin (2017) imposes the closely related NCC $\operatorname{cov}_{t}\left(M_{t+1} R_{t+1}, R_{t+1}\right) \leq 0$. The two conditions are plausible for similar reasons: in any reasonable model, $M_{t+1}$ is negatively correlated with the return on the market, $R_{t+1}$, and we know from the bound of Hansen and Jagannathan (1991), coupled with the empirical fact that high Sharpe ratios are available, that $M_{t+1}$ is highly volatile.

In fact, the two conditions are equivalent in the lognormal case. Suppose that the SDF $M_{t+1}$ and return $R_{t+1}$ are conditionally jointly lognormal and write $r_{f, t+1}=\log R_{f, t+1}, \mu_{t}=\log \mathbb{E}_{t} R_{t+1}$, and $\sigma_{t}^{2}=\operatorname{var}_{t} \log R_{t+1}$. Then the mNCC and NCC are both equivalent to the assumption that the conditional Sharpe ratio of the asset, $\lambda_{t} \equiv\left(\mu_{t}-r_{f, t+1}\right) / \sigma_{t}$, exceeds its conditional volatility, $\sigma_{t}{ }^{13}$

The Sharpe ratio of the market is typically thought of as being on the order of $30 \%$ to $50 \%$, while the volatility of the market is on the order of $16 \%$ to $20 \%$. Thus the mNCC holds in the calibrated models of Campbell and Cochrane (1999), Bansal and Yaron (2004), Bansal et al. (2014), and Campbell et al.

\footnotetext{
${ }^{12}$ In fact, it is so minor that the distinction between options on $P_{t+1}$ and options on $P_{t+1}+D_{t+1}$ is often neglected entirely in the literature. For example, Neuberger (2012) "avoid[s] irrelevant complications with interest rates and dividends" by treating options on forward prices as observable, as do Schneider and Trojani (2019), and (essentially equivalently) Carr and Wu (2009) use options on stocks as proxies for options on stock futures. These authors effectively assume that our inequality (27) holds with equality.

${ }^{13}$ By Stein's lemma, $\operatorname{cov}_{t}\left(M_{t+1} R_{t+1}, \log R_{t+1}\right)=\operatorname{cov}_{t}\left(\log M_{t+1}+\log R_{t+1}, \log R_{t+1}\right)$. By $\operatorname{lognor-}$ mality of $M_{t+1}$ and $R_{t+1}$, the fact that $\mathbb{E}_{t}\left(M_{t+1} R_{t+1}\right)=1$ is equivalent to $\log \mathbb{E}_{t} M_{t+1}+\log \mathbb{E}_{t} R_{t+1}=$ $-\operatorname{cov}_{t}\left(\log M_{t+1}, \log R_{t+1}\right)$. It follows from these two observations that $\operatorname{cov}_{t}\left(M_{t+1} R_{t+1}, \log R_{t+1}\right) \leq 0$ if and only if $\operatorname{var}_{t} \log R_{t+1} \leq \log \mathbb{E}_{t} R_{t+1}-r_{f, t+1}$, that is, if and only if $\lambda_{t} \geq \sigma_{t}$. Under lognormality, this condition is equivalent to $\operatorname{cov}_{t}\left(M_{t+1} R_{t+1}, R_{t+1}\right) \leq 0$, as shown by Martin (2017).
} 
(2018), among many others. But Martin (2017) argues that option prices are inconsistent with the lognormality assumption. This motivates the following result, which provides a sufficient condition for the mNCC to hold without requiring lognormality.

RESULT 4: Suppose that an investor's SDF takes the form

$$
M_{t+1}=\beta \frac{V_{W}\left(W_{t+1}, z_{t+1}\right)}{V_{W}\left(W_{t}, z_{t}\right)},
$$

where $V_{W}$ is the investor's marginal value of wealth, and $z_{t}$ is a vector of state variables, with signs chosen so that $V_{W}$ is weakly decreasing in each (just as it is decreasing in $W_{t+1}$ ). We allow time $t+1$ wealth, $W_{t+1}$, to be invested in the market and in some other asset or portfolio of assets with gross return $\widetilde{R}_{t+1}$ :

$$
W_{t+1}=\underbrace{\alpha_{t}\left(W_{t}-C_{t}\right) R_{t+1}}_{\text {market wealth, } W_{m, t+1}}+\underbrace{\left(1-\alpha_{t}\right)\left(W_{t}-C_{t}\right) \widetilde{R}_{t+1}}_{\text {nonmarket wealth }} \text {, where } \alpha_{t} \in(0,1] \text {. }
$$

If (i) $R_{t+1}, \widetilde{R}_{t+1}$, and the elements of $z_{t+1}$ are associated random variables, ${ }^{14}$ (ii) the investor ensures that the share of wealth in the market, $W_{m, t+1} / W_{t+1}$, is at least $\theta \in(0,1]$, some fixed constant, and (iii) the investor's relative risk aversion $-W V_{W W} / V_{W}$ (which need not be constant) is at least $1 / \theta$, then the mNCC holds.

Proof: We must show that $\operatorname{cov}_{t}\left(-R_{t+1} V_{W}\left(W_{t+1}, z_{t+1}\right), \log R_{t+1}\right) \geq 0$, that is, we must prove that the covariance of two functions of $R_{t+1}, \widetilde{R}_{t+1}$, and $z_{t+1}$ is positive. The two functions are

$$
f\left(R_{t+1}, \widetilde{R}_{t+1}, z_{t+1}\right)=-R_{t+1} V_{W}\left(\alpha_{t}\left(W_{t}-C_{t}\right) R_{t+1}+\left(1-\alpha_{t}\right)\left(W_{t}-C_{t}\right) \widetilde{R}_{t+1}, z_{t+1}\right)
$$

and

$$
g\left(R_{t+1}, \widetilde{R}_{t+1}, z_{t+1}\right)=\log R_{t+1} .
$$

(As the covariance is conditional on time $t$ information, we can treat $\alpha_{t}$ and $W_{t}-C_{t}$ as known constants.) As the random variables are associated, the result follows if $f$ and $g$ are each weakly increasing functions of their arguments. The assumptions above ensure that this is the case. For example, differentiating $f$ with respect to $R_{t+1}$, we need $-V_{W}\left(W_{t+1}, z_{t+1}\right)-\alpha_{t}\left(W_{t}-\right.$ $\left.C_{t}\right) R_{t+1} V_{W W}\left(W_{t+1}, z_{t+1}\right) \geq 0$ or, rearranging,

$$
-\frac{W_{t+1} V_{W W}\left(W_{t+1}, z_{t+1}\right)}{V_{W}\left(W_{t+1}, z_{t+1}\right.} \geq \frac{W_{t+1}}{W_{m, t+1}} .
$$

\footnotetext{
14 The concept of associated random variables (Esary, Proschan, and Walkup (1967)) extends the concept of nonnegative correlation in a manner that can be extended to the multivariate setting. Jointly Normal random variables are associated if and only if they are nonnegatively correlated (Pitt (1982)), and increasing functions of associated random variables are associated. It follows that lognormal random variables are associated if and only if they are nonnegatively correlated.
} 
But this holds, by assumptions (ii) and (iii):

$$
-\frac{W_{t+1} V_{W W}\left(W_{t+1}, z_{t+1}\right)}{V_{W}\left(W_{t+1}, z_{t+1}\right.} \stackrel{\text { (iii) }}{\geq} \frac{1}{\theta} \stackrel{\text { (ii) }}{\geq} \frac{W_{t+1}}{W_{m, t+1}} .
$$

It is immediate that $f$ and $g$ are weakly increasing in their other arguments.

Result 4 provides a flexible set of conditions under which the mNCC holds.

EXAMPLE 1: Suppose that there is a representative investor who maximizes utility over next-period wealth and who chooses to invest her wealth fully in the stock market. Then by Result 4, the mNCC holds so long as her relative risk aversion (which need not be constant) is at least one at all levels of wealth. Furthermore, if the representative investor has log utility, then the mNCC is tight-that is, the inequality holds with equality-because $M_{t+1} R_{t+1}=1$ is a constant.

EXAMPLE 2: Alternatively, if the investor keeps at least (say) a third of her wealth in the market, then her relative risk aversion must be at least three. We also require that the market and nonmarket returns be associated. In the lognormal case, this holds if the market and nonmarket returns are nonnegatively correlated.

These examples make no assumption about the beliefs of other investors in the economy. We can therefore think from the perspective of a rational investor surrounded by other investors, some of whom are potentially irrational. We think that the assumption that the investor chooses to invest fully in the stock market represents a natural benchmark in such cases, but the possibility arises that the lower bound might be violated-say in the late 1990s-because no rational investor would want to hold the market. We discuss this possibility after introducing the sentiment measure in Section IV. We provide further examples of situations in which the mNCC holds in Appendix B.

We can now state our lower bound on expected log returns.

RESUlt 5: Suppose Assumptions 1 and 2 hold. Denote by call $_{t}(K)$ and $\operatorname{put}_{t}(K)$ the time $t$ prices of call and put options on $P_{t+1}$ with strike $K$, and denote by $F_{t}$ the time $t$ forward price of the index for settlement at time $t+1$. Then we have

$$
\mathbb{E}_{t} r_{t+1}-r_{f, t+1} \geq \underbrace{\frac{1}{P_{t}}\left\{\int_{0}^{F_{t}} \frac{\operatorname{put}_{t}(K)}{K} d K+\int_{F_{t}}^{\infty} \frac{\operatorname{call}_{t}(K)}{K} d K\right\}}_{\text {LVIX }} .
$$


Proof: As $\mathbb{E}_{t}^{*} R_{t+1}=R_{f, t+1}$ and $\mathbb{E}_{t}^{*} P_{t+1}=F_{t}$, the inequality (27) can be rearranged as

$$
\frac{1}{R_{f, t+1}} \mathbb{E}_{t}^{*} R_{t+1} \log R_{t+1}-\log R_{f, t+1} \geq \frac{1}{R_{f, t+1}}\left[\mathbb{E}_{t}^{*}\left(\frac{P_{t+1}}{P_{t}} \log \frac{P_{t+1}}{P_{t}}\right)-\frac{F_{t}}{P_{t}} \log \frac{F_{t}}{P_{t}}\right]
$$

The right-hand side of this inequality can be measured directly from option prices using a result of Breeden and Litzenberger (1978) that can be rewritten, following Carr and Madan (2001), to give, for any sufficiently well-behaved function $g(\cdot)$,

$$
\frac{1}{R_{f, t+1}}\left[\mathbb{E}_{t}^{*} g\left(P_{t+1}\right)-g\left(\mathbb{E}_{t}^{*} P_{t+1}\right)\right]=\int_{0}^{F_{t}} g^{\prime \prime}(K) \operatorname{put}_{t}(K) d K+\int_{F_{t}}^{\infty} g^{\prime \prime}(K) \operatorname{call}_{t}(K) d K .
$$

Setting $g(x)=\frac{x}{P_{t}} \log \frac{x}{P_{t}}$, we have $g^{\prime \prime}(x)=1 /\left(P_{t} x\right)$. Thus,

$$
\frac{1}{R_{f, t+1}}\left[\mathbb{E}_{t}^{*}\left(\frac{P_{t+1}}{P_{t}} \log \frac{P_{t+1}}{P_{t}}\right)-\frac{F_{t}}{P_{t}} \log \frac{F_{t}}{P_{t}}\right]=\frac{1}{P_{t}}\left\{\int_{0}^{F_{t}} \frac{\text { put }_{t}(K)}{K} d K+\int_{F_{t}}^{\infty} \frac{\operatorname{call}_{t}(K)}{K} d K\right\} .
$$

The result follows by combining the identity (26), the inequalities (28) and (30), and equation (31).

We refer to the right-hand side of equation (29) as LVIX because it is reminiscent of the definition of the VIX index, which, in our notation, is

$$
\mathrm{VIX}_{t}^{2}=2 R_{f, t+1}\left\{\int_{0}^{F_{t}} \frac{\operatorname{put}_{t}(K)}{K^{2}} d K+\int_{F_{t}}^{\infty} \frac{\operatorname{call}_{t}(K)}{K^{2}} d K\right\}
$$

and of the SVIX index introduced by Martin (2017),

$$
\operatorname{SVIX}_{t}^{2}=\frac{2}{R_{f, t+1} P_{t}^{2}}\left\{\int_{0}^{F_{t}} \operatorname{put}_{t}(K) d K+\int_{F_{t}}^{\infty} \operatorname{call}_{t}(K) d K\right\}
$$

We do not annualize our definition (29), so to avoid unnecessary clutter we also do not annualize the definitions of VIX and SVIX above. We typically choose the period length from $t$ to $t+1$ to be 12 months. The forecasting horizon dictates the maturity of the options, so we use options expiring in 12 months to measure expectations of 12-month log returns.

VIX, SVIX, and LVIX place differing weights on option prices. VIX has weighting function $1 / K^{2}$ on the prices of options with strike $K$, LVIX has weighting function $1 / K$, and SVIX has a constant weighting function. In this sense, we can think of LVIX as lying halfway between VIX and SVIX. (We could also introduce a factor of two into the definition of LVIX to make the indices look even more similar to one another, but have chosen not to.) 


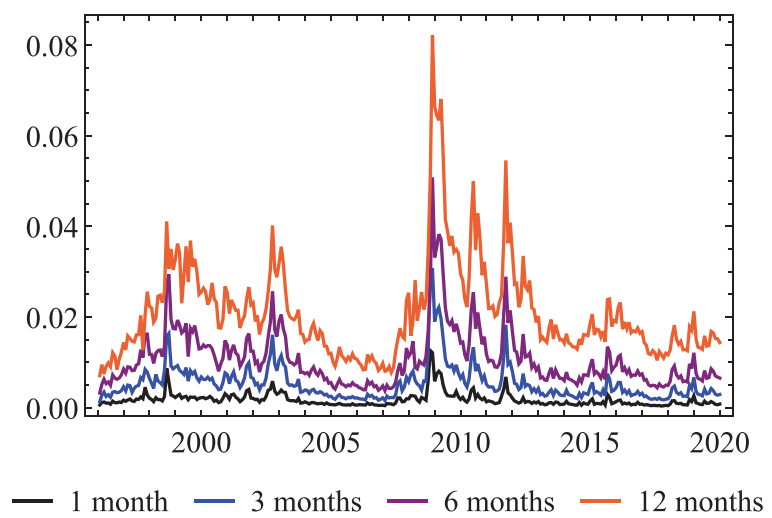

Figure 1. This figure plots the LVIX index, which provides a lower bound on the market's expected excess log return $\mathbf{E}_{t} \boldsymbol{r}_{\boldsymbol{t}+1}-\boldsymbol{r}_{\boldsymbol{f}, \boldsymbol{t}+1}$, by Result 5. (Color figure can be viewed at wileyonlinelibrary.com)

We calculate LVIX using end-of-month interest rates and S\&P 500 index option (mid) prices from OptionMetrics. In practice, we do not observe option prices at all strikes between zero and infinity, so we have to truncate the integral on the right-hand side of (29) (as does the CBOE in its calculation of the VIX index). In doing so, we understate the idealized value of the integral. That is, our lower bound would be even higher if given perfect data, and thus it is conservative.

Figure 1 plots LVIX $_{t}$, at the end of each month, over our sample period from January 1996 to June 2019. Under our maintained assumptions, the large spikes visible during in 2008 to 2009 , for example, indicate that expected excess log returns were very high in the depths of the subprime crisis, consistent with the results of Martin (2017). Of greater relevance for this paper, expected excess log returns were also relatively high around the turn of the millennium, despite the high valuation ratios that prevailed at the time.

One might worry that option markets were illiquid, or segmented from the broader stock market, during the late 1990s. Lamont and Thaler (2003) present evidence that this was indeed the case for certain individual stocks (most famously for options on Palm), and ascribe the anomalous behavior of prices of these stocks, and of options on the stocks, to the difficulty or impossibility of shorting the stocks. As short-selling the broader stock market was possible at low cost throughout this period (for example, via the futures market), we do not expect this to be an issue for our approach. But to address the more general concern that option markets may have exhibited extreme bid-ask spreads at the time, we recompute the LVIX index using bid prices as opposed to mid prices. (We use bid rather than ask prices to be conservative, as this will drive our sentiment indicator down.) As shown in Figure 2, doing so has very little effect on our results. 


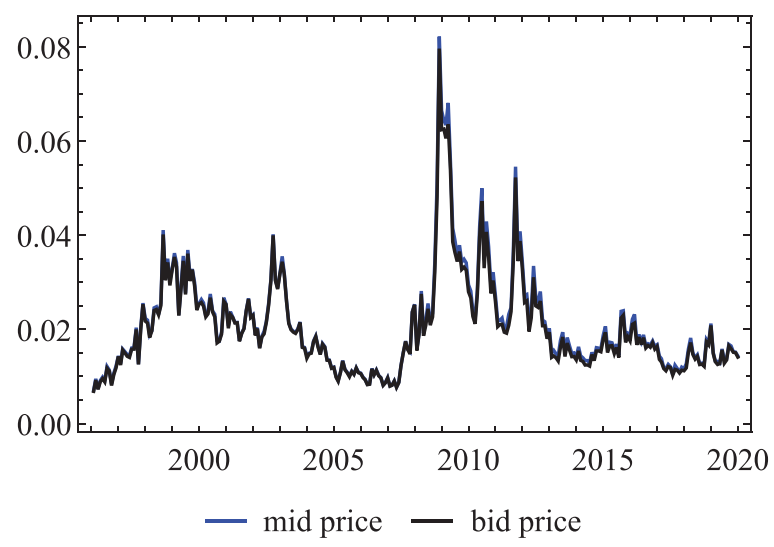

Figure 2. LVIX $_{t}$, S\&P 500 index, annual horizon, computed using mid prices or bid prices. (Color figure can be viewed at wileyonlinelibrary.com)

\section{A. Empirical Evidence on the Modified NCC}

We motivated the inequality of Result 5 via a theoretical argument that the $\mathrm{mNCC}$ should hold. We can also assess the inequality empirically by examining the realized forecast errors $r_{t+1}-r_{f, t+1}-\mathrm{LVIX}_{t}$. To do so, we carry out a one-sided $t$-test of the hypothesis that inequality (29) fails. Using a block bootstrap, ${ }^{15}$ we find a $p$-value of 0.097 . Thus, despite the relatively short sample period imposed on us by the availability of option price data, we can reject the hypothesis with moderate confidence. This supports our approach.

More optimistically, it is natural to ask whether inequality (29) might approximately hold with equality (though we emphasize that this does not need to be the case for our approach to make sense). For this to be true, we would need both (27) and (28) to hold with approximate equality. As the conditional volatility of dividends is substantially lower than that of prices, it is reasonable to think that this is indeed the case for (27), and as noted in footnote 12, much of the literature implicitly makes that assumption. Meanwhile the mNCC (28) would hold with equality if (but not only if) one adopts the perspective of an investor with log utility who chooses to hold the market, as is clear from the proof provided in Example 2 above. The perspective of such an investor has been shown to provide a useful benchmark for forecasting returns on the stock market (Martin (2017)), on individual stocks (Martin and Wagner (2019)), and on currencies (Kremens and Martin (2019)).

Table VIII reports results from the regression

$$
r_{t+1}-r_{f, t+1}=\alpha+\beta \times \operatorname{LVIX}_{t}+\varepsilon_{t+1}
$$

\footnotetext{
${ }^{15}$ As our sample is 276 months long, we use a block length of seven months, following the $T^{1 / 3}$ rule of thumb for block length for a sample of length $T$. For comparison, the corresponding $p$-value using naive OLS standard errors would be 0.001 .
} 
Table VIII

Coefficient Estimates for Regression (32), 96:01 to 17:12

\begin{tabular}{lccccc}
\hline Horizon & $\widehat{\alpha}$ & s.e. & $\widehat{\beta}$ & s.e. & $R^{2}$ \\
\hline $3 \mathrm{~m}$ & 0.009 & {$[0.018]$} & 1.381 & {$[3.629]$} & $0.54 \%$ \\
$6 \mathrm{~m}$ & -0.004 & {$[0.021]$} & 3.128 & {$[1.514]$} & $3.67 \%$ \\
$9 \mathrm{~m}$ & -0.002 & {$[0.041]$} & 2.948 & {$[1.439]$} & $3.70 \%$ \\
$12 \mathrm{~m}$ & 0.006 & {$[0.063]$} & 2.493 & {$[1.613]$} & $2.86 \%$ \\
\hline
\end{tabular}

at horizons of three, six, nine, and 12 months. Returns are computed by compounding the CRSP monthly gross return of the S\&P 500. We report HansenHodrick (1980) standard errors to allow for heteroskedasticity and for autocorrelation that arises due to overlapping observations. If inequality (29) holds with equality, we should find $\alpha=0$ and $\beta=1$. We do not reject this hypothesis at any horizon; and at the six- and nine-month horizons, we can reject the hypothesis that $\beta=0$ at conventional significance levels.

\section{A Sentiment Indicator}

We now adopt the perspective of a hypothetical investor whose expectations and SDF satisfy the mNCC so that the lower bound (29) of Section III applies. We also assume that this hypothetical investor's beliefs are consistent with the predictive relationship (25) between valuation ratios, returns, and dividend growth, as studied in Section II. We do so to force the investor's beliefs to be consistent with the historical evidence, in order to prevent him or her from "explaining" asset prices simply by concluding that "this time is different" (in the words of Reinhart and Rogoff (2009)).

We can derive a lower bound on such an investor's subjective expectations about fundamentals by subtracting $\mathbb{E}_{t}\left(r_{t+1}-g_{t+1}\right)$, as revealed by valuation ratios, from $\mathbb{E}_{t} r_{t+1}$, as revealed by interest rates and option prices:

$$
\begin{aligned}
\mathbb{E}_{t} g_{t+1} & =r_{f, t+1}+\mathbb{E}_{t}\left(r_{t+1}-r_{f, t+1}\right)-\mathbb{E}_{t}\left(r_{t+1}-g_{t+1}\right) \\
& \geq r_{f, t+1}+\operatorname{LVIX}_{t}-\mathbb{E}_{t}\left(r_{t+1}-g_{t+1}\right) .
\end{aligned}
$$

The inequality follows (under our maintained Assumptions 1 and 2) because $\mathbb{E}_{t} r_{t+1}-r_{f, t+1} \geq$ LVIX $_{t}$, as shown in Result 5 .

We use $y_{t}$ to measure $\mathbb{E}_{t}\left(r_{t+1}-g_{t+1}\right)$ via the fitted value $\widehat{a}_{0}+\widehat{a}_{1} y_{t}$, as in Table I, giving the sentiment indicator $B_{t}$, which is a lower bound on the investor's expected log dividend growth:

$$
B_{t}=r_{f, t+1}+\frac{1}{P_{t}}\left[\int_{0}^{F_{t}} \frac{\operatorname{put}_{t}(K)}{K} d K+\int_{F_{t}}^{\infty} \frac{\operatorname{call}_{t}(K)}{K} d K\right]-\left(\widehat{a}_{0}+\widehat{a}_{1} y_{t}\right)
$$

We estimate the coefficients $\widehat{a}_{0}$ and $\widehat{a}_{1}$ using an expanding window: at time $t$ they are estimated using data from 1947 until time $t$. As a result, $B_{t}$ is observable at time $t$. 


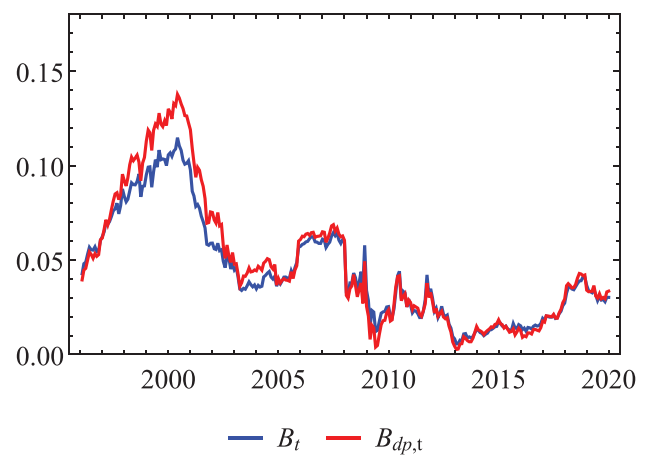

Panel A. Full sample

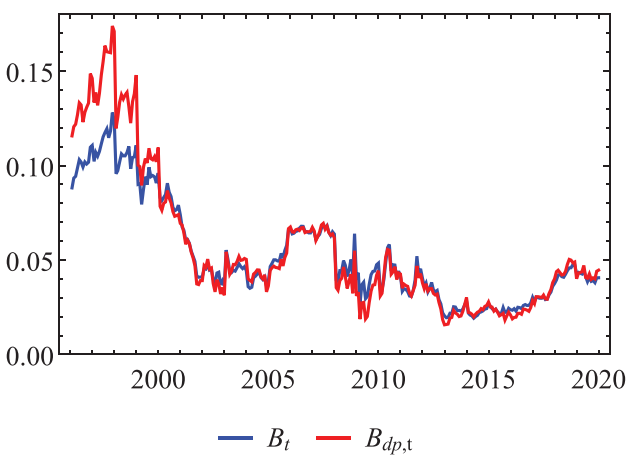

Panel B. Real time

Figure 3. The sentiment indicator, computed using the full sample to estimate the relationship between $y_{t}$ and $\boldsymbol{r}_{t+1}-\boldsymbol{g}_{\boldsymbol{t}+1}$ (left) or using an expanding window (right). (Color figure can be viewed at wileyonlinelibrary.com)

As we have discussed, $B_{t}$ can be interpreted as a lower bound on expected dividend growth, $\mathbb{E}_{t} g_{t+1}$. If $\mathbb{E}_{t} g_{t+1}$ itself follows an $\mathrm{AR}(1)$-as in the work of Bansal and Yaron (2004) and many others-then $B_{t}$ can also be interpreted, after rescaling, as a lower bound on long-run dividend expectations. The reason is that if $\mathbb{E}_{t+1} g_{t+2}-\bar{g}=\phi_{g}\left(\mathbb{E}_{t} g_{t+1}-\bar{g}\right)+\varepsilon_{g, t+1}$, then we can define a measure of expected long-run dividend growth, at time $t$, as

$$
(1-\rho) \sum_{i \geq 0} \rho^{i}\left(\mathbb{E}_{t} g_{t+1+i}-\bar{g}\right)=\frac{1-\rho}{1-\rho \phi_{g}}\left(\mathbb{E}_{t} g_{t+1}-\bar{g}\right) .
$$

(We have introduced a factor $1-\rho$ so that long-run expected dividend growth can be interpreted as a weighted average of all future periods' expected growth because the weights $(1-\rho) \rho^{i}$ sum to one.)

Figure 3, Panel A plots $B_{t}$ over our sample period using the full sample from 1947 to 2019 to estimate the relationship between $y_{t}\left(\right.$ or $\left.d p_{t}\right)$ and $r_{t+1}-g_{t+1}$. We work at an annual horizon, ${ }^{16}$ so the value of $B_{t}$ at a given point in time is (subject to our maintained assumptions) a lower bound on the expected dividend growth over the subsequent year. Figure 3, Panel B shows the corresponding results using an expanding window to estimate the relationship, so that the resulting series is observable in real time. Encouragingly, the indicator behaves stably as we move from full-sample information to real-time information. Unless otherwise indicated, henceforth we work with the series that is observable in real time.

The figures also show modified indicators, $B_{d p, t}$, that use $d p_{t}$ rather than $y_{t}$ to measure $\mathbb{E}_{t}\left(r_{t+1}-g_{t+1}\right)$, as in (23). These have the advantage of familiarity$d p_{t}$ has been widely used in the literature-but the disadvantage that they

\footnotetext{
${ }^{16}$ We find similar results at the two-year horizon, as can be seen from Tables IA.I and IA.II, and Figures IA. 1 and IA.2, of the Internet Appendix. The Internet Appendix is available in the online version of the article on The Journal of Finance website.
} 


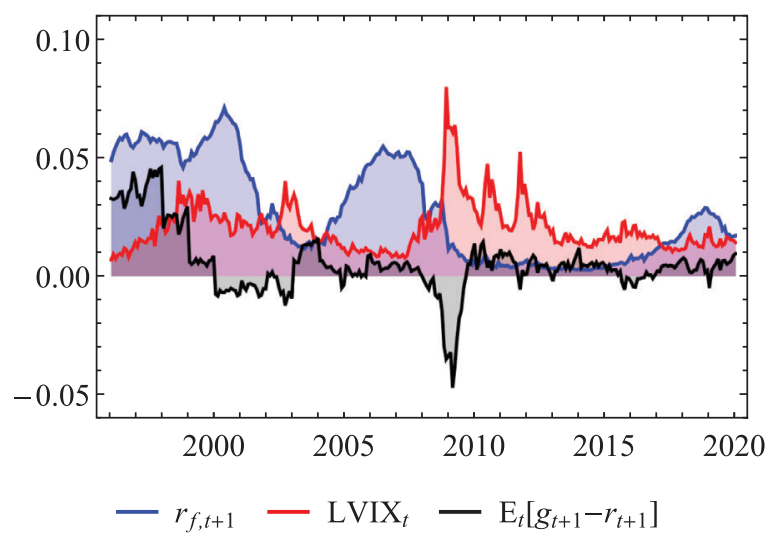

Figure 4. The three components of the sentiment indicator. (Color figure can be viewed at wileyonlinelibrary.com)

may err on the side of signaling a bubble too soon, as shown in Result 3. Consistent with this prediction, the two series line up fairly closely, but the $B_{d p, t}$ series are less conservative-in that they suggest even higher $\mathbb{E}_{t} g_{t+1}$-during the period in the late 1990s when valuation ratios were far from their mean.

Note, moreover, that net dividend growth satisfies $\mathbb{E}_{t} \frac{D_{t+1}}{D_{t}}-1>\mathbb{E}_{t} g_{t+1}$ because $e^{g_{t+1}}-1>g_{t+1}$. Our lower bound on expected log dividend growth therefore implies even higher expected arithmetic dividend growth. If dividend growth were conditionally lognormal, for example, we would have $\log \mathbb{E}_{t} \frac{D_{t+1}}{D_{t}}=$ $\mathbb{E}_{t} g_{t+1}+\frac{1}{2} \operatorname{var}_{t} g_{t+1}$. The variance term is small unconditionally-in our sample period, $\operatorname{varg}_{t+1} \approx 0.005$-but it is plausible that during the late 1990s there was unusually high uncertainty about log dividend growth.

Figure 4 plots the three components of the sentiment indicator $B_{t}$ from 1996 to 2019. LVIX and $\mathbb{E}_{t}\left(g_{t+1}-r_{t+1}\right)$ moved in opposite directions for most of our sample period, with high valuation ratios occurring at times of low-risk premia. But all three components were above their sample means during the late 1990s.

In particular, our approach implies that the expected annual log dividend growth perceived by our hypothetical representative investor rose above $12 \%$ around the turn of the millennium, a degree of optimism that we do not think was reasonable. If we reject this conclusion, we must reject at least one of the assumptions that delivered it.

The first possibility is that there is no investor whose preferences and beliefs are such that the mNCC is satisfied. In particular, this would be a violation of the equilibrium models and various examples discussed in Section III.

Alternatively, if the mNCC did hold, then-for the hypothetical investor to perceive high expected log returns and, simultaneously, low expected log dividend growth during the bubble period-he or she must have believed that the historical forecasting relationship between dividend yield and $\mathbb{E}_{t}\left(r_{t+1}-g_{t+1}\right)$ 
had broken down, perhaps because of a "paradigm shift" or because the predictive coefficients estimated using historical data failed to reflect the true population values. ("This time is different!") To see this, write $\widehat{\mathbb{E}}_{t}\left(r_{t+1}-g_{t+1}\right)$ for the regression-implied time $t$ forecast of $r_{t+1}-g_{t+1}$, which we now allow to differ from the agent's forecast $\mathbb{E}_{t}\left(r_{t+1}-g_{t+1}\right)$. Then, from inequality (33), we have

$$
\begin{aligned}
\mathbb{E}_{t} g_{t+1}= & r_{f, t+1}+\mathbb{E}_{t}\left(r_{t+1}-r_{f, t+1}\right)-\widehat{\mathbb{E}}_{t}\left(r_{t+1}-g_{t+1}\right) \\
& +\left[\widehat{\mathbb{E}}_{t}\left(r_{t+1}-g_{t+1}\right)-\mathbb{E}_{t}\left(r_{t+1}-g_{t+1}\right)\right] \\
& \geq \underbrace{r_{f, t+1}+\operatorname{LVIX}_{t}-\widehat{a}_{0}-\widehat{a}_{1} y_{t}}_{B_{t}}+\left[\widehat{\mathbb{E}}_{t}\left(r_{t+1}-g_{t+1}\right)-\mathbb{E}_{t}\left(r_{t+1}-g_{t+1}\right)\right] .
\end{aligned}
$$

An agent who believed, in the late $1990 \mathrm{~s}$, that $\mathbb{E}_{t} g_{t+1}$ was lower than $B_{t}$ must therefore have concluded that $\widehat{\mathbb{E}}_{t}\left(r_{t+1}-g_{t+1}\right)<\mathbb{E}_{t}\left(r_{t+1}-g_{t+1}\right)$. By the loglinearization (24), this is equivalent to $\mathbb{E}_{t} y_{t+1}<\widehat{\mathbb{E}}_{t} y_{t+1}$. On this interpretation, our hypothetical investor's beliefs were consistent only because she expected $y_{t+1}$ to remain, in the short run, lower-and valuations higher-than suggested by the historical evidence. We discuss this possibility further in Section IV.B.1 below.

\section{A. Alternative Stochastic Processes for $y_{t}$}

We have modeled $y_{t}$ as following an $\operatorname{AR}(1)$ to avoid overfitting. Aside from the obvious advantages of parsimony, the partial autocorrelations of $y_{t}$, as shown in Figure C.1 of Appendix C, support this choice: the partial autocorrelations of $y_{t}$ at lags greater than one are close to zero.

The question of how to model $y_{t}$ is not central to the point of this paper, however, so we also consider the possibility that $y_{t}$ follows an $\operatorname{AR}(2)$ or $\operatorname{AR}(3)$. If $y_{t}$ follows an $\operatorname{AR}(2)$ process, then from the linearization (24), we have

$$
r_{t+1}-g_{t+1}=\alpha+\beta y_{t}+\gamma y_{t-1}+\varepsilon_{t+1},
$$

while if $y_{t}$ follows an $\mathrm{AR}(3)$ process, we have

$$
r_{t+1}-g_{t+1}=\alpha+\beta y_{t}+\gamma y_{t-1}+\delta y_{t-2}+\varepsilon_{t+1} \text {. }
$$

The results of these regressions are reported in Table A.II of Appendix A.

The corresponding lower bounds on $\mathbb{E}_{t} g_{t+1}$ are shown in Figure 5 . They are very similar to our baseline measure during the late $1990 \mathrm{~s}$, but they are lower during the crisis of 2008 to 2009 and higher in its aftermath. Once again, we note that the indicator behaves fairly stably as we move from full-sample information to real-time information. Figure IA.3 in the Internet Appendix plots the minimum of the three series computed under $\operatorname{AR}(1), \operatorname{AR}(2)$, and $A R(3)$ assumptions; this serves as a conservative lower bound. 


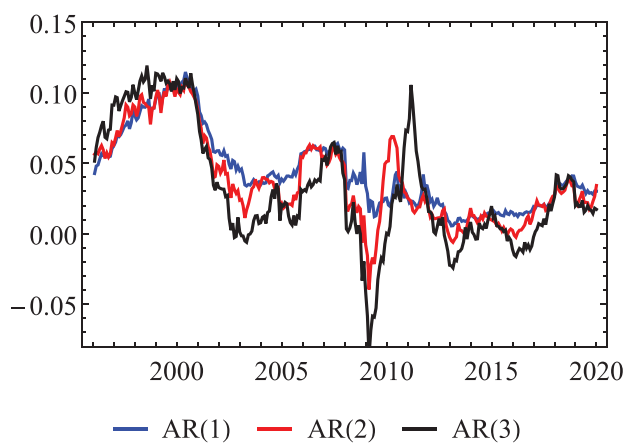

Panel A. Full sample

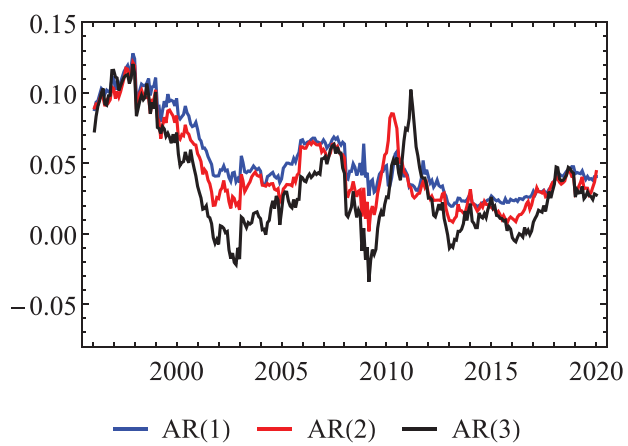

Panel B. Real time

Figure 5. Sentiment indicators calculated on a full-sample or real-time basis, assuming that $y_{t}$ follows an $\mathbf{A R}(1), \mathbf{A R}(2)$, or $\mathbf{A R}(3)$ process. (Color figure can be viewed at wileyonlinelibrary.com)

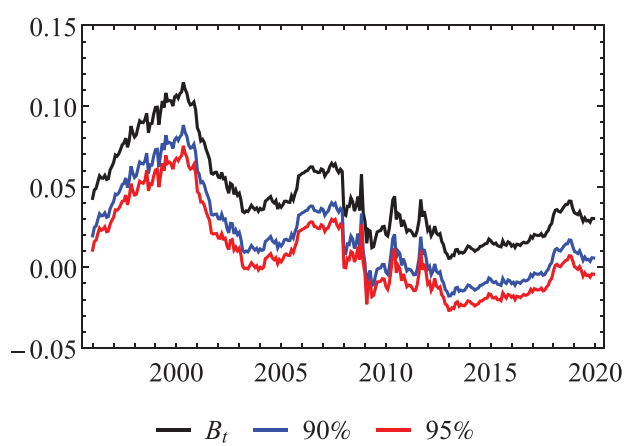

Panel A. AR(1), full sample

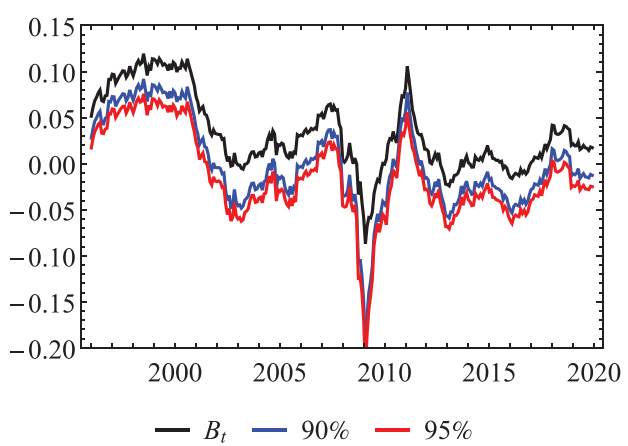

Panel B. AR(3), full sample

Figure 6. $B_{t}$ with one-sided confidence interval. (Color figure can be viewed at wileyonlinelibrary.com)

\section{B. Variations}

\section{B.1. Estimation Uncertainty}

The coefficients in the regression of $r_{t+1}-g_{t+1}$ onto $y_{t}$ (and its lags, in the $\mathrm{AR}(2)$ and $\mathrm{AR}(3)$ cases) are estimated with statistical uncertainty. To illustrate, Figure 6 plots block-bootstrapped one-sided $90 \%$ and $95 \%$ confidence intervals for our baseline measure $B_{t} .{ }^{17}$ At the edge of the $90 \%$ (95\%) confidence

\footnotetext{
${ }^{17}$ We compute the confidence intervals, at each point in time $t$, by adding $r_{f, t}+\operatorname{LVIX}_{t}$ to the bottom $5 \%$ (or $10 \%$ ) quantile of the bootstrapped sample of $\mathbb{E}_{t}\left[g_{t+1}-r_{t+1}\right]$. In the case of the $\mathrm{AR}(1)$ model, for example, the bootstrapped sample is created by repeating the following procedure 10,000 times (i.e., for $k=1, \ldots, 10,000$ ): (i) estimate $\widehat{a}_{k}$ and $\widehat{b}_{k}$ via OLS from the equation $r_{k, t+1}-g_{k, t+1}=a_{k}+b_{k} y_{k, t}+\varepsilon_{k, t+1}$, where the sample of $\left(r_{k, t+1}, g_{k, t+1}, y_{k, t}\right)$ is drawn in blocks of length $T^{1 / 3}$, with replacement, from the annual data of $\left(r_{t+1}, g_{t+1}, y_{t}\right)$ between 1947 and 2019, and (ii) compute $-\widehat{a}_{k}-\widehat{b}_{k} y_{t}$, where $y_{t}$ is the monthly series between 1996:01 and 2019:06.
} 
intervals, the lower bounds on expected dividend growth peak at $9.3 \%(7.9 \%)$ for the AR(1) model and at 9.5\% (7.8\%) for the AR(3) model.

While we think these numbers remain implausibly high, one might perhaps argue that they were reasonable forecasts of expected dividend growth. We emphasize, however, that when using the $90 \%$ or $95 \%$ percentile as the estimate of expected dividend growth, the implicit position taken is that the historical relationship between valuation ratios and $r_{t}-g_{t}$-as embodied in the point estimates, the correct central measure-is misleading. Furthermore, a prudent policymaker should also entertain the symmetric possibility that in the presence of estimation uncertainty, the true $B_{t}$ is substantially higher than implied by the central point estimates of the predictive coefficients.

\section{B.2. What If the Valuation Ratio Follows a Random Walk?}

A true believer in the New Economy might have argued that our measure of $\mathbb{E}_{t}\left(r_{t+1}-g_{t+1}\right)$, which is based on an assumption that $y_{t}$ follows an $\mathrm{AR}(1)$ - or $\mathrm{AR}(2)$ or AR(3) — had broken down during the late 1990s. Perhaps the most aggressive possibility our hypothetical investor could reasonably entertain is the "random walk" view that the price-dividend ratio had entirely ceased to meanrevert, as considered by Campbell $(2008,2018)$. Such a perspective might also be adopted by a cautious central banker to justify inaction on the basis that valuation ratios could remain very high indefinitely. ${ }^{18}$

We now show how to accommodate this possibility. If $y_{t}$ follows a random walk, then, from equation (14),

$$
\mathbb{E}_{t} g_{t+1} \stackrel{(14)}{=} \mathbb{E}_{t} r_{t+1}-y_{t} \geq \underbrace{\operatorname{LVIX}_{t}+r_{f, t}-y_{t}}_{\widetilde{B}_{t}},
$$

where we define a variant on our previous indicator,

$$
\widetilde{B}_{t}=\operatorname{LVIX}_{t}+r_{f, t}-y_{t}
$$

that has the further benefit of not requiring estimation of any free parameters. More generally, if all one knows is that $\mathbb{E}_{t} y_{t+1} \geq y_{t}$-irrespective of the details of the evolution of $y_{t}$-then equation (24) implies that

$$
\mathbb{E}_{t} g_{t+1} \geq \widetilde{B}_{t}
$$

Figure 7 plots the time series of $\widetilde{B}_{t}$. Even if valuation ratios were expected to follow a random walk in the late $1990 \mathrm{~s}-\mathrm{a}$ dubious proposition in any casethe implied expectations about cash flow growth appear implausibly high.

Unlike our preferred indicator, $B_{t}$, the random walk version $\widetilde{B}_{t}$ spiked almost as high during the subprime crisis as it did around the turn of the millennium. This pattern reflects the fact that implied volatility, and hence the LVIX index, rose dramatically during the last months of 2008 , indicating that log returns

\footnotetext{
${ }^{18}$ It can also be interpreted as a conservative approach to addressing Stambaugh bias.
} 


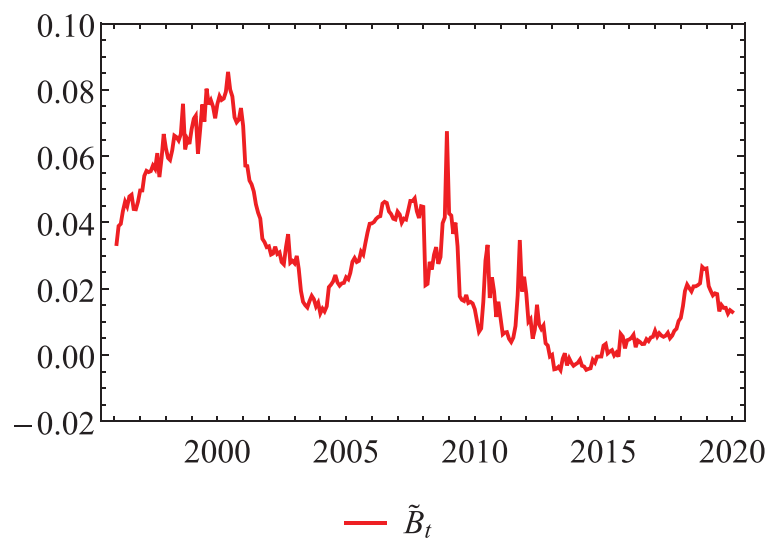

Figure 7. The alternative sentiment indicator, $\widetilde{B}_{\boldsymbol{t}}$. (Color figure can be viewed at wileyonlinelibrary.com)

were expected to be very high over the subsequent year (by Result 5). From the perspective of our notional policymaker who believed that valuation ratios follow a random walk, these high expected log returns could only have reflected high expected log dividend growth. This prediction is unreasonable, in our view, because the random walk assumption is unreasonable. The point is that even a policymaker who believed valuation ratios followed a random walk would have had to perceive unusually high expected dividend growth in the late 1990s.

\section{B.3. What If Dividend Growth Is Unforecastable?}

If dividend growth is unforecastable (in the sense that $\mathbb{E}_{t} g_{t+k}=\bar{g}$ for $k \geq 1$, as in the work of Campbell and Cochrane (1999) and many others), then valuation ratios reveal long-run expectations of log returns while LVIX reveals the corresponding short-run expectations.

Specifically, if dividend growth is unforecastable and $y_{t}$ is stationary, then from equation (15)

$$
y_{t}=(1-\rho) \sum_{i=0}^{\infty} \rho^{i} \mathbb{E}_{t}\left[r_{t+1+i}-g_{t+1+i}\right]=(1-\rho) \mathbb{E}_{t}\left[r_{t+1}\right]+(1-\rho) \rho \sum_{i=0}^{\infty} \rho^{i} \mathbb{E}_{t}\left[r_{t+2+i}\right]-\bar{g}
$$

This equation can be rearranged to give

$$
\mathbb{E}_{t} r_{t+1}-(1-\rho) \sum_{i \geq 0} \rho^{i} \mathbb{E}_{t} r_{t+2+i}=\frac{\mathbb{E}_{t} r_{t+1}-y_{t}-\bar{g}}{\rho}
$$


Exploiting the inequality $\mathbb{E}_{t} r_{t+1}-r_{f, t} \geq \operatorname{LVIX}_{t}$ of Result 5 , we can conclude that

$$
\underbrace{\mathbb{E}_{t} r_{t+1}}_{\text {short-run returns }}-\underbrace{(1-\rho) \sum_{i \geq 0} \rho^{i} \mathbb{E}_{t} r_{t+2+i}}_{\text {long-run returns }} \geq \frac{\operatorname{LVIX}_{t}+r_{f, t}-y_{t}-\bar{g}}{\rho}=\frac{\widetilde{B}_{t}-\bar{g}}{\rho} .
$$

This inequality provides an alternative interpretation of the indicator $\widetilde{B}_{t}=$ LVIX $_{t}+r_{f, t}-y_{t}$ that we define in equation (34) above and that is plotted in Figure 7. If dividend growth is unforecastable, then unusually high levels of $\widetilde{B}_{t}$ indicate that short-run expected log returns are unusually high relative to subsequent long-run expected log returns.

\section{B.4. Are Valuation Ratios Alone Enough?}

Valuation ratios alone would make for an even simpler sentiment indicator. Are they enough? In theory, no: as we have argued, valuation ratios can be high for good reasons if interest rates are low or if risk premia are low (and are widely understood to be low), or both, and our measure embraces this fact by incorporating $r_{f, t}$ and $\mathrm{LVIX}_{t}$.

Nonetheless, theory aside, we do know of course that valuation ratios were very high during the late $1990 \mathrm{~s}$, so it is interesting from a purely empirical perspective to see how they compare with $B_{t}$. We plot the valuation ratio measures $-y_{t}$ and $p d_{t}$ on the same axes as $B_{t}$ over our sample period in Figure C.2 in Appendix C. For ease of comparison, we standardize all three series to have zero mean and unit standard deviation and we use the full-sample version of $B_{t}$ so that the predictive coefficients do not vary over the time series. The sentiment index $B_{t}$ gives a clearer indication of bubbliness in the market at the start of our sample, from 1996 to 2000, in the sense that it is generally around onehalf to one standard deviation further above its mean than are the valuation ratio series.

In the opposite direction, valuation ratios have been very high in recent years. But our measure suggests that this does not represent a bubble, as the high valuation ratios have reflected unusually low interest rates (and, for much of this period, low volatility).

\section{B.5. Can the Methodology be Applied in Other Markets?}

Our approach can be applied to other assets if their returns obey the mNCC. It is reasonable to expect that this is the case for stock market indices, for example. Figure 8 plots our sentiment index for the NASDAQ-100. To do so, we calculate LVIX using the mid prices of NASDAQ-100 options from OptionMetrics and estimate the predictive regression (25) over the period 1983 to 2019 using NASDAQ-100 dividend yield and price data from Datastream. (As the predictive regression is estimated over a shorter time series, we present results using the full sample rather than using an expanding window.) The 


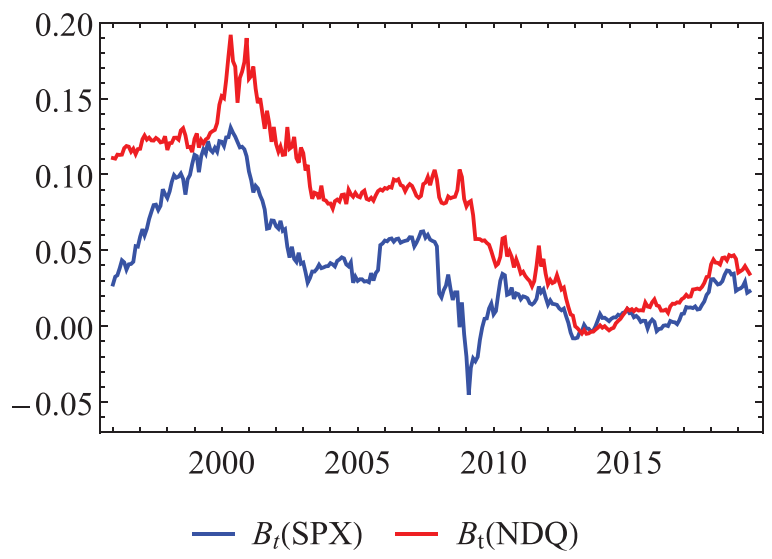

Figure 8. The sentiment index computed for the NASDAQ-100 index (and, for comparison, the baseline sentiment index for the S\&P 500). (Color figure can be viewed at wileyonlinelibrary.com)

sentiment index for the NASDAQ-100 was substantially higher than that for the S\&P 500 around and before the turn of the millennium, consistent with the conventional view that sentiment was particularly elevated in tech stocks at the time.

For "hedge" assets, such as gold, one would expect the direction of the inequality (28) to be reversed. This rules out using our approach to detect bubbles in such assets. The situation is more promising in the case of individual stocks: it may be possible to argue that the mNCC holds for stocks with betas sufficiently close to or greater than one, along the lines of Martin and Wagner (2019) and Kadan and Tang (2019), but we leave this extension for future research.

\section{B.6. Nonlinearity in the Functional Form}

We can also allow for a nonlinear relationship between $r_{t+1}-g_{t+1}$ and $y_{t}$. In Appendix A, we report the results of regressions of the form

$$
r_{t+1}-g_{t+1}=a_{0}+a_{1} y_{t}+a_{2} y_{t}^{2}+\varepsilon_{t+1}
$$

and

$$
r_{t+1}-g_{t+1}=a_{0}+a_{1} y_{t}+a_{2} y_{t}^{2}+a_{3} y_{t}^{3}+\varepsilon_{t+1} .
$$

Figure 9, Panel A, shows that these regressions deliver similar results to the linear specification reported above when we use the full sample period to estimate the coefficients $a_{i}$. But the coefficient estimates in the higher-order specifications are strikingly unstable when we estimate the regressions in real time using expanding windows (Figure 9, Panel B), even though the regressions are 

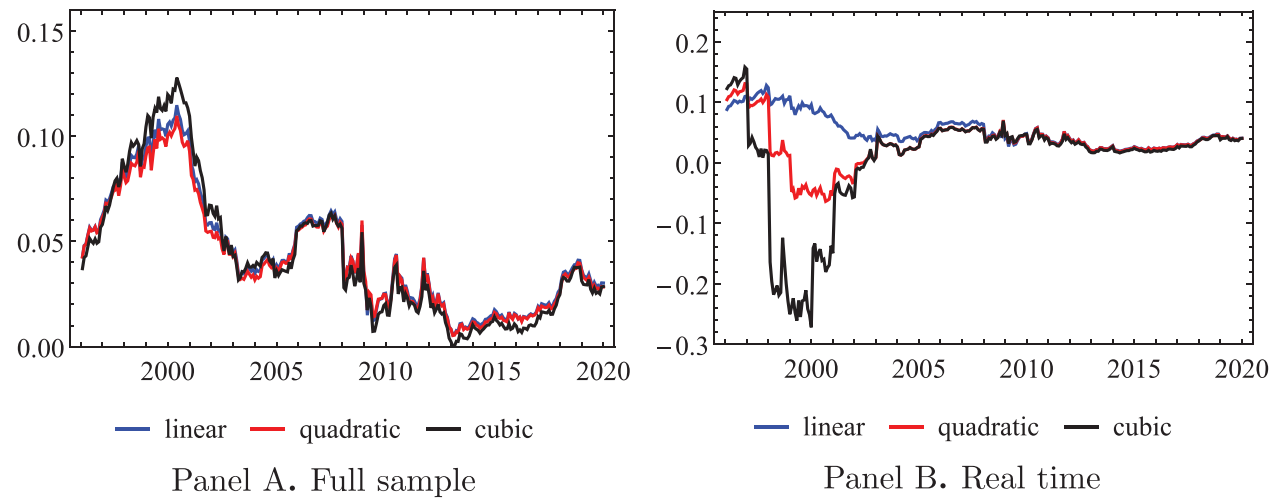

Figure 9. Sentiment indicators calculated with linear, quadratic, and cubic specifications for the relationship between expected $\boldsymbol{r}_{t+1}-\boldsymbol{g}_{\boldsymbol{t}+1}$ and $\boldsymbol{y}_{\boldsymbol{t}}$ (Color figure can be viewed at wileyonlinelibrary.com)

estimated on almost 50 years of data at the start of our sample period, that is, on data from 1947 to 1996.

In the late $1990 \mathrm{~s}$, for example, the estimated cubic specification implies a negative relationship between $y_{t}$ and forecast $r_{t+1}-g_{t+1}$ around the thenprevailing value of $y_{t}$. That is, given the then-recent association of unusually low dividend yield with high realized returns, the cubic specification predicts extremely high returns going forward, as shown in Figure C.3, Panel A, in Appendix C. (It is important that the low dividend yields at the time were unusual, because the cubic specification makes it possible to associate high returns with extremely low yields without materially altering the longestablished relationship between low returns and low yields that prevails over the usual range of yields.)

We view this exercise as a cautionary tale. Given that bubbles occur fairly rarely, it is particularly important to avoid the possibility that an (over-)elaborate model achieves superior performance in-sample by overfitting the historical data. The ingredients of a bubble indicator should behave stably during historically unusual periods, as our simple linear specification does (Figure C.3, Panel B in Appendix C).

\section{Other Indicators of Market Conditions}

We now compare the sentiment indicator to other indicators of financial conditions that have been proposed in the literature. We standardize all time series to have zero mean and unit standard deviation throughout this section, for ease of comparison and so that correlations can be equivalently interpreted as betas (noting that $\operatorname{corr}(X, Y)=\frac{\operatorname{cov}(X, Y)}{\operatorname{var} X}$ if $X$ and $Y$ have unit standard deviation). 


\section{A. Volume}

We start by exploring the relationship with volume, which has been widely proposed as a signature of bubbles (see, for example, Harrison and Kreps (1978), Duffie, Garleanu, and Pedersen (2002), Cochrane (2003), Lamont and Thaler (2003), Ofek and Richardson (2003), Scheinkman and Xiong (2003), Hong, Scheinkman, and Xiong (2006), Barberis et al. (2018)). We construct a daily measure of volume using Compustat data from January 1983 to December 2017 by summing the product of shares traded and daily low price over all S\&P 500 stocks on each day. (We find essentially identical results if we use daily high prices to construct the measure.) As volume trended strongly upward during our sample period, we subtract a linear trend from log volume. We do so using an expanding window, so that our detrended log volume measure, which we call $v_{t}$, is (like $B_{t}$ ) observable at time $t$.

The left panel of Figure 10 plots detrended log volume, $v_{t}$, and $B_{t}$ over the sample period, with both series standardized to zero mean and unit variance. There is a remarkable similarity between the two series, so it is worth emphasizing that they are each based on entirely different input data. The sentiment index is a leading indicator of volume: the right panel shows the correlation between $B_{t+k}$ and $v_{t}$, where $k$ is measured in months. The shaded area indicates a block bootstrapped ${ }^{19} 95 \%$ confidence interval. The correlation between the two is higher than 0.9 when $k$ is around -10 months. Thus, Figure 10 shows that $B_{t-10}$ is highly statistically significant as a forecaster of $v_{t}$ (and, to a lesser extent, that $v_{t}$ is a statistically significant forecaster of $B_{t+10}$ ).

\section{B. Survey Expectations of Long-Term Earnings Growth}

We next compare $B_{t}$ to a quarterly time series of financial analysts' longterm earnings growth (LTG) forecasts constructed by Nagel and Xu (2019). Figure 11 plots the LTG series against $B_{t}$, with the latter computed as in our baseline measure (i.e., using an $\mathrm{AR}(1)$ and with an expanding window to compute predictive coefficients) and with an $\mathrm{AR}(3)$ using full-sample data. There is a striking similarity between the two series-particularly when the sentiment indicator is computed using an $\mathrm{AR}(3)$ and full-sample estimation of the predictive regression-but we note that $B_{t}$ rose more rapidly during the late $1990 \mathrm{~s}$.

\section{The Probability of a Crash}

One expects that the probability of a crash should be higher during a bubble episode; if not, the episode is perhaps not actually a bubble. ${ }^{20}$ To assess this prediction, we use a measure of the (time $t$ conditional) probability of a crash derived by Martin (2017, Result 2), which can be computed in terms of option

\footnotetext{
${ }^{19}$ As before, we draw 10,000 bootstrap samples, using block length of $T^{1 / 3}$. We use the same procedure in the correlation plots shown in Figures 11, 12, C.4, C.5, and IA.4 to IA.10.

${ }^{20}$ Greenwood, Shleifer, and You (2019) document, at the industry level, that sharp increases in stock prices do indeed signal a heightened probability of a crash.
} 


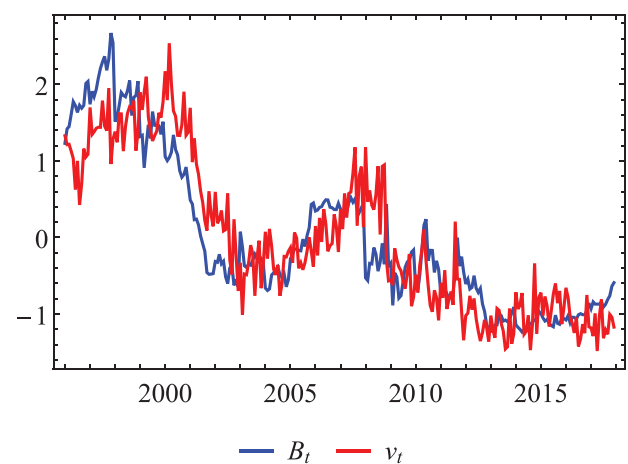

Panel A. $B_{t}$ and $v_{t}$

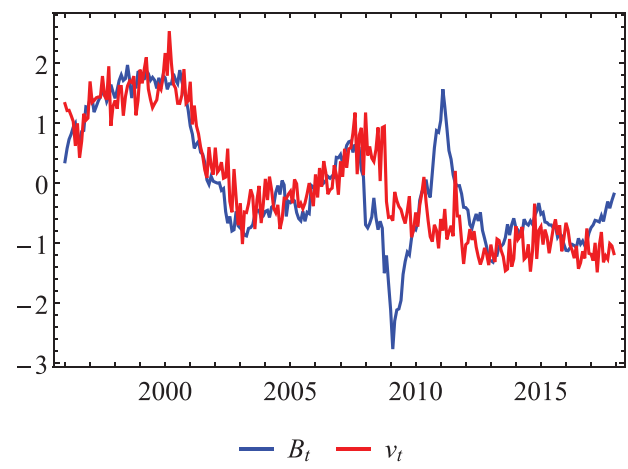

Panel C. $B_{t}\left(\mathrm{AR}(3)\right.$, full sample) and $v_{t}$

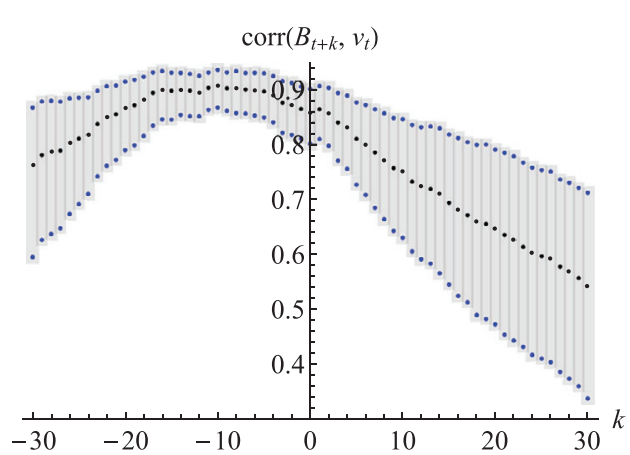

Panel B. Correlation between $B_{t+k}$ and $v_{t}$

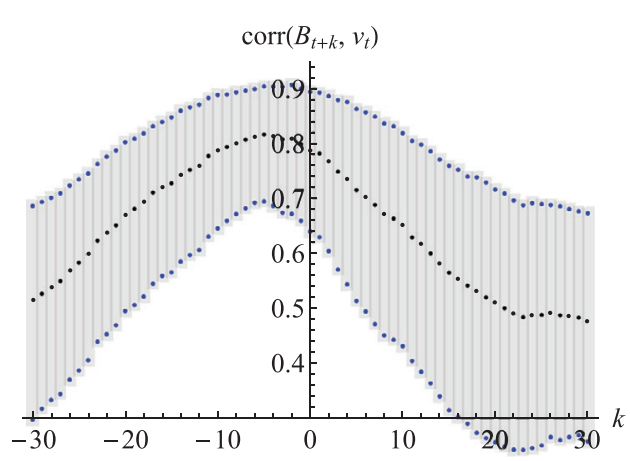

Panel D. Correlation between $B_{t+k}$ (AR(3), full sample) and $v_{t}$

Figure 10. The sentiment indicator and detrended log volume (standardized). Shaded areas in the right panel indicate bootstrapped $95 \%$ confidence intervals. $k$ is measured in months. (Color figure can be viewed at wileyonlinelibrary.com)

prices as

$$
\mathbb{P}_{t}\left(R_{t+1}<\alpha\right)=\alpha\left[\operatorname{put}_{t}^{\prime}\left(\alpha P_{t}\right)-\frac{\operatorname{put}_{t}\left(\alpha P_{t}\right)}{\alpha P_{t}}\right],
$$

where $\operatorname{put}_{t}^{\prime}(K)$ is the first derivative of the put price as a function of strike, evaluated at $K$. This measure represents the probability of a market decline perceived by an unconstrained log investor who chooses to hold the market. We also require that the investor is marginal in option markets, so we therefore rule out the possibility that these markets are segmented from the stock market. (In other words, the above calculation relies on a stronger assumption than the rest of the paper, namely, that the SDF $M_{t+1}$ satisfies $M_{t+1}=1 / R_{t+1}$; this implies that the mNCC holds with equality.) The probability of a crash (35) is high when out-of-the-money put prices are highly convex, as a function of strike, at strikes at and below $\alpha P_{t}$. By contrast, the measure of volatility (29) 


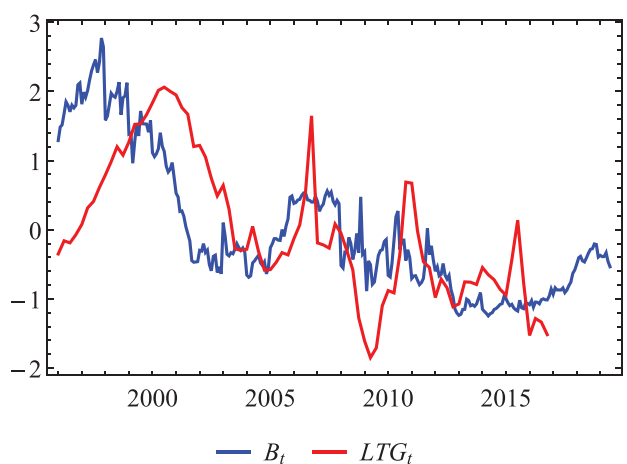

Panel A. $B_{t}$ and LTG

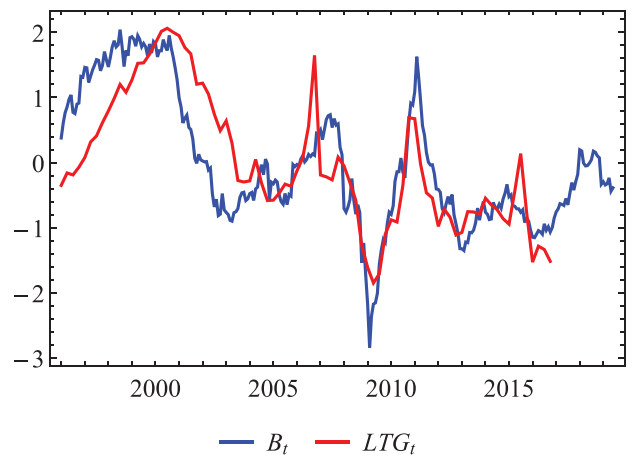

Panel C. $B_{t}(\mathrm{AR}(3)$, full sample) and LTG

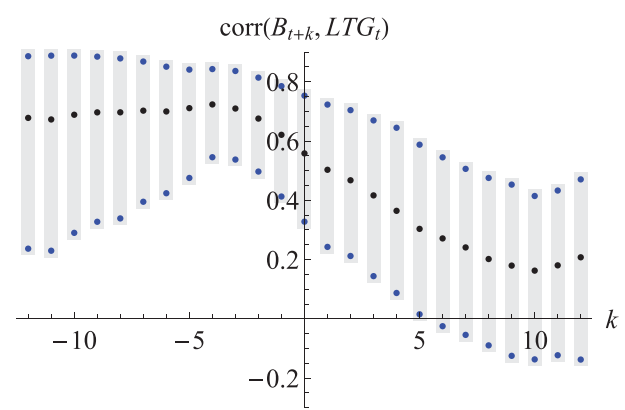

Panel B. $\operatorname{corr}\left(B_{t+k}, \mathrm{LTG}_{t}\right)$

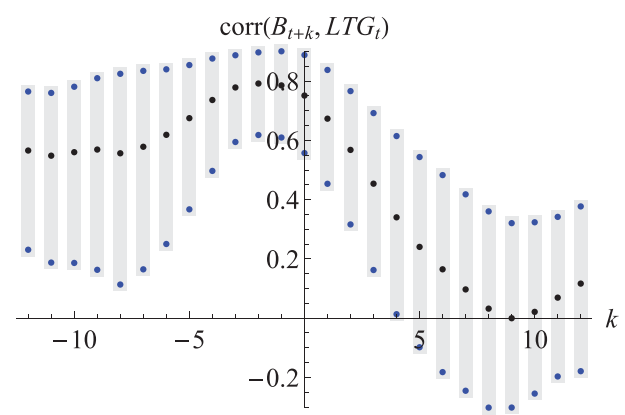

Panel D. $\operatorname{corr}\left(B_{t+k}, \mathrm{LTG}_{t}\right) \quad(\mathrm{AR}(3)$, full sample)

Figure 11. $B_{\boldsymbol{t}}$ plotted against the LTG measure of Nagel and Xu (2019). All series are standardized. As LTG is quarterly, $k$ is measured in quarters. (Color figure can be viewed at wileyonlinelibrary.com)

that is relevant for our sentiment indicator is a function of option prices across the full range of strikes of out-of-the-money puts and calls.

The left panel of Figure 12 plots the crash probability over time. The probability of a crash was elevated during the late 1990s, consistent with standard intuition about bubbles. But it was also high in the aftermath of the subprime crisis, an episode that we would certainly not identify as bubbly. The right panel shows the correlation between the two series at different leads and lags. The sentiment measure is a leading indicator of crash probability at horizons of about two years.

The possibility that high valuation ratios, expected log returns, and expected log dividend growth can coexist with a high crash probability (in the mind of our representative investor) is reminiscent of the view of the world colorfully articulated by former Citigroup chief executive Chuck Prince in a July 2007 interview with the Financial Times: "When the music stops, in terms of 


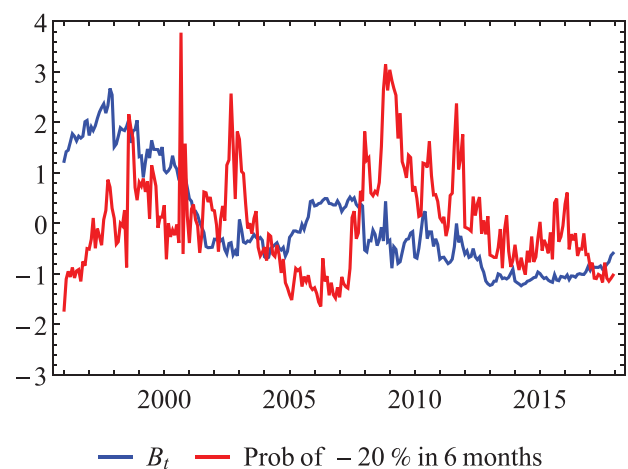

Panel A. $B_{t}$ and crash probability

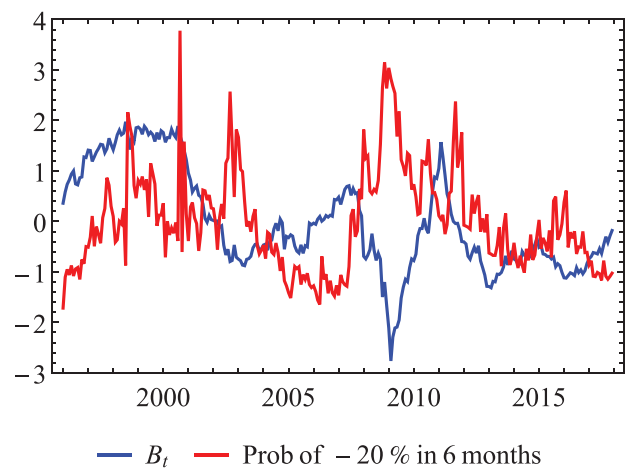

Panel C. $B_{t}(\mathrm{AR}(3)$, full-sample) and crash probability

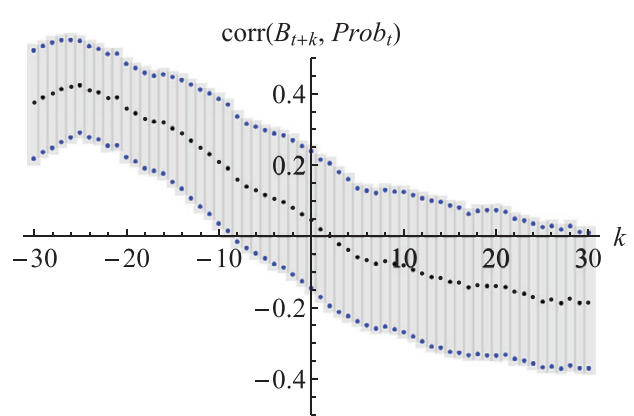

Panel B. Correlation between $B_{t+k}$ and crash probability

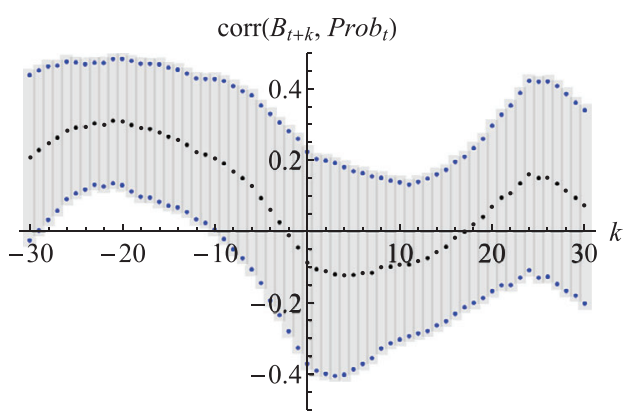

Panel D. Correlation between $B_{t+k}$ $(\mathrm{AR}(3)$, full-sample) and crash probability

Figure 12. The sentiment indicator, volume, and crash probability. Shaded areas in the right-hand panels indicate bootstrapped $95 \%$ confidence intervals. $k$ is measured in months. (Color figure can be viewed at wileyonlinelibrary.com)

liquidity, things will be complicated. But as long as the music is playing, you've got to get up and dance. We're still dancing."

\section{Other Measures}

The panels of Figures C.4 and C.5 compare the sentiment index to various other measures of financial conditions: the excess bond premium (EBP) of Gilchrist and Zakrajšek (2012), the National Financial Conditions Index (NFCI), and the Adjusted National Financial Conditions Index (ANFCI), all of which are generated on a weekly basis by the Federal Reserve Bank of Chicago. We convert them to monthly series by taking the last week's observation in each calendar month. 
Lastly, Figures IA. 4 through IA.9 in the Internet Appendix compare $B_{t}$ with various series drawn from quarterly surveys studied by Ben-David, Graham, and Harvey (2013). The variables $E R_{1 \mathrm{yr}}$ and $E R_{10 \mathrm{yr}}$ are, respectively, the crosssectional average subjective expectations of stock market returns over one- and 10-year horizons, as reported by survey respondents, $E E R_{1 \mathrm{yr}}$ and $E E R_{10 \mathrm{yr}}$ are the corresponding average subjective expected excess returns, and ERstd $d_{1 \mathrm{yr}}$ and $E R s t d_{10 \mathrm{yr}}$ are disagreement measures at the same horizons (that is, the cross-sectional standard deviations of reported subjective expected returns). Figure IA.10 compares $B_{t}$ with a quarterly time series of average subjective expectations of dividend growth constructed by De la O and Myers (2021). The measures of mean subjective expected returns and of mean subjective expected dividend growth are positively correlated with $B_{t}$, whereas the measures of mean subjective expected excess returns and of disagreement are negatively correlated with $B_{t}$. We are hesitant to draw firm conclusions from this evidence, however, as the comparison series do not include the period of greatest interest, from 1996 to 2000 .

\section{Conclusion}

We present a sentiment indicator based on interest rates, index option prices, and the market valuation ratio. The indicator can be interpreted as a lower bound on the expected dividend growth that must be perceived by an unconstrained, rational investor with risk aversion equal to at least one who is happy to invest his or her wealth fully in the stock market and whose beliefs are consistent with historical evidence on the relationship between valuation ratios, returns, and dividend growth.

The bound was very high during the late 1990s, reflecting dividend growth expectations that in our view were unreasonably optimistic-hence our description of it as a sentiment indicator-and that were not realized ex post. We also show that it is a leading indicator of detrended volume, of LTG expectations, and of various measures of stress in the financial system.

In simple terms, we characterize the late 1990 s as a bubble because valuation ratios and short-run expected returns-as revealed by interest rates and our LVIX measure-were simultaneously high. Both aspects are important. We would not view high valuation ratios at a time of low expected returns, or low valuation ratios at a time of high expected returns, as indicative of a bubble. On the contrary, the latter scenario occurred in the aftermath of the market crash in 2008.

Our measure does not point to an unreasonable level of market sentiment at the end of our sample period in 2019 , as it interprets the high valuation ratios that prevailed at the time as being justified by the low levels of interest rates and of implied volatility.

Volatility and valuation ratios have, of course, long been linked to bubbles. A novel feature of our approach is that we use some theory to motivate our definitions of volatility and of valuation ratios, and to make the link quantitative. There are various choices to be made regarding the construction of the 
indicator. We make these choices in a conservative way to avoid "crying bubble" prematurely, in the hope that the indicator might be useful to cautious policymakers in practice. Our approach does ultimately require an appeal to the good judgment of policymakers, as we do not address the hard question of how to identify whether a given level of expected dividend growth is reasonable. We do not see a way to avoid some degree of expert judgment in identifying market-wide bubbles, but we believe that the approach proposed in this paper would make it easier for such judgment to be applied in a focussed and disciplined manner.

\section{Initial submission: August 30, 2019; Accepted: February 4, 2021 \\ Editors: Stefan Nagel, Philip Bond, Amit Seru, and Wei Xiong}

\section{Appendix A: Other Specifications}

If $y_{t}$ follows an $\mathrm{AR}(1)$ with autocorrelation $\phi$, then the linear approximation (24) reduces to

$$
\mathbb{E}_{t}\left(r_{t+1}-g_{t+1}\right)=\underbrace{\frac{\rho(\phi-1)}{1-\rho} \bar{y}}_{\alpha}+\underbrace{\frac{1-\rho \phi}{1-\rho}}_{\beta} y_{t} .
$$

In the body of the paper, we estimate the predictive relationship between $r_{t+1}-g_{t+1}$ and the predictor variable $y_{t}$ (and $d p_{t}$ ) via linear regression. Under our AR(1) assumption, we could also estimate the constant term and the coefficient on $y_{t}$ directly, as in (A1), by estimating $\rho$ and the autocorrelation $\phi$. Table A.I shows that both approaches give similar results.

Alternatively, if $y_{t}$ follows an $\operatorname{AR}(2)$ process, then from the linearization (24), we have

$$
r_{t+1}-g_{t+1}=\alpha+\beta y_{t}+\gamma y_{t-1}+\varepsilon_{t+1},
$$

while if $y_{t}$ follows an $\operatorname{AR}(3)$ process, we have

$$
r_{t+1}-g_{t+1}=\alpha+\beta y_{t}+\gamma y_{t-1}+\delta y_{t-2}+\varepsilon_{t+1} .
$$

Table A.II reports the results of these regressions.

We can also consider the effect of allowing for quadratic or cubic functional relationships between $r_{t+1}-g_{t+1}$ and $y_{t}$. Table A.III reports results from the

Table A.I

Comparison of $A R(1)$ Parameterization and Linear Regression

\begin{tabular}{lccc}
\hline Method & $\alpha$ & $\beta$ & $R^{2}$ \\
\hline OLS & -0.07 & 3.54 & $8.33 \%$ \\
$A R(1)$ & -0.08 & 3.81 & $8.51 \%$ \\
\hline
\end{tabular}

Annual price and dividend data, 1947 to 2019, from CRSP (cash reinvestment), as in Table I. 
Table A.II

Predictive Regressions for S\&P 500, Annual Data, Cash Reinvestment, 1947 to 2017

\begin{tabular}{|c|c|c|c|c|c|c|c|c|c|}
\hline & $\widehat{a}$ & s.e. & $\widehat{\beta}$ & s.e. & $\widehat{\gamma}$ & s.e. & $\widehat{\delta}$ & s.e. & $R^{2}$ \\
\hline $\mathrm{AR}(1)$ & -0.067 & [0.049] & 3.415 & [1.317] & & & & & $7.73 \%$ \\
\hline $\mathrm{AR}(2)$ & -0.056 & [0.053] & 6.098 & [3.378] & -2.991 & [3.339] & & & $8.84 \%$ \\
\hline $\mathrm{AR}(3)$ & -0.040 & {$[0.055]$} & 6.473 & [3.313] & 0.651 & [3.231] & -4.457 & [2.373] & $11.32 \%$ \\
\hline
\end{tabular}

Table A.III

Predictive Regressions for S\&P 500, Annual Data, Cash Reinvestment, 1947 to 2017

\begin{tabular}{lccccccccc}
\hline & $\widehat{a}_{0}$ & s.e. & $\widehat{a}_{1}$ & s.e. & $\widehat{a}_{2}$ & s.e. & $\widehat{a}_{3}$ & s.e. & $R^{2}$ \\
\hline linear & -0.067 & {$[0.049]$} & 3.415 & {$[1.317]$} & & & & & $7.73 \%$ \\
quadratic & -0.072 & {$[0.111]$} & 3.740 & {$[6.348]$} & -4.390 & {$[81.250]$} & & $7.73 \%$ \\
cubic & -0.120 & {$[0.230]$} & 9.350 & {$[20.960]$} & -166.400 & {$[576.000]$} & 1402.800 & {$[4821.300]$} & $7.81 \%$ \\
\hline
\end{tabular}

regressions

$$
r_{t+1}-g_{t+1}=a_{0}+a_{1} y_{t}+a_{2} y_{t}^{2}+\varepsilon_{t+1}
$$

and

$$
r_{t+1}-g_{t+1}=a_{0}+a_{1} y_{t}+a_{2} y_{t}^{2}+a_{3} y_{t}^{3}+\varepsilon_{t+1} .
$$

\section{Appendix B: Further Examples}

We provide some other illustrations of situations in which the mNCC holds. These illustrations are intended as proof-of-concept rather than as fully fleshed-out models, so we have simplified them as far as possible.

EXAMPLE B1 (Heterogeneous preferences): This example is a simplification of Longstaff and Wang (2012), except that we do not need to make any assumptions on the distribution of aggregate consumption growth. Consider a two-period economy with complete markets and two agents with homogeneous beliefs and power utility, but with differing coefficients of risk aversion, $\gamma_{2}>\gamma_{1} \geq 1$. Agent $i$ 's problem is therefore

$$
\max \frac{C_{i, t}^{1-\gamma_{i}}}{1-\gamma_{i}}+\beta \mathbb{E}_{t} \frac{C_{i, t+1}^{1-\gamma_{i}}}{1-\gamma_{i}}
$$

As markets are complete and beliefs are homogeneous, the SDF is unique, so that $\beta\left(C_{1, t+1} / C_{1, t}\right)^{-\gamma_{1}}=\beta\left(C_{2, t+1} / C_{2, t}\right)^{-\gamma_{2}}$. Following Longstaff and Wang (2012) in assuming that $\gamma_{1}=\gamma$ and $\gamma_{2}=2 \gamma$ to ensure a closed-form solution, we 
therefore have

$$
\frac{C_{1, t+1}}{C_{1, t}}=\left(\frac{C_{2, t+1}}{C_{2, t}}\right)^{2}
$$

Writing $Y_{t}=C_{1, t}+C_{2, t}$ for aggregate consumption, this implies that

$$
C_{2, t+1}=\frac{2}{a}\left(\sqrt{1+a Y_{t+1}}-1\right),
$$

where the constant $a=4 C_{1, t} / C_{2, t}^{2}$ reflects the relative wealth of the two agents.

We wish to check whether the mNCC holds for the return on the market, that is, the aggregate consumption claim. To do so, we construct a representative agent for whom the mNCC holds. (Although agents 1 and 2 are not representative-neither invests only in the market-they have the same beliefs and SDF as the representative agent, so it will then follow that the mNCC holds for them too.) In the usual way, the representative agent consumes $Y_{t+1}$ and has marginal utility $v^{\prime}\left(Y_{t+1}\right)$ that is proportional to $C_{2, t+1}^{-2 \gamma}$. Integrating, the representative agent's utility function is

$$
v\left(Y_{t+1}\right)=\frac{\left(\sqrt{1+a Y_{t+1}}-1\right)^{2(1-\gamma)}}{2(1-\gamma)}+\frac{\left(\sqrt{1+a Y_{t+1}}-1\right)^{1-2 \gamma}}{1-2 \gamma} .
$$

The representative agent's relative risk aversion is therefore low in good times and high in bad times, and it lies between $\gamma$ and $2 \gamma$ :

$$
-\frac{Y_{t+1} v^{\prime \prime}\left(Y_{t+1}\right)}{v^{\prime}\left(Y_{t+1}\right)}=\gamma+\frac{\gamma}{\sqrt{1+a Y_{t+1}}} \rightarrow\left\{\begin{array}{ll}
\gamma & \text { as } Y_{t+1} \rightarrow \infty \\
2 \gamma & \text { as } Y_{t+1} \rightarrow 0
\end{array} .\right.
$$

As $\gamma \geq 1$, the mNCC holds.

ExAmple B2 (Heterogeneous beliefs): This example is based on Martin and Papadimitriou (2021). A continuum of investors with log utility over terminal wealth trade a risky asset in unit supply ("the market") and a riskless asset in zero net supply. The net riskless rate is zero. Uncertainty evolves on a binomial tree, so the risky asset's return, $R$, equals $R_{u}$ at the up-node and $R_{d}$ at the down-node; we choose labels so that $R_{u}>R_{d}$. Investors, indexed by $h \in(0,1)$, have heterogeneous beliefs: investor $h$ believes that the probability of an upmove is $h$. On wealth-weighted average, investors must hold one unit of the asset to clear the market. At any node, we can define a representative agent $H \in(0,1)$ who invests fully in the risky asset with no borrowing or lending. We also define the risk-neutral probability of an up-move (on which all investors agree) as $p^{*} \in(0,1)$. Optimists $(h>H)$ lever up, while sufficiently pessimistic investors $\left(h<p^{*}\right)$ go short, as they perceive that the market earns a negative risk premium.

These assumptions imply that the representative agent perceives the market as growth-optimal, and hence that $\frac{H}{R_{u}}+\frac{1-H}{R_{d}}=1$ (as the gross riskless rate is one). However, again using the fact that the gross riskless rate equals one, 
we must also have $p^{*} R_{u}+\left(1-p^{*}\right) R_{d}=1$ by the defining property of the riskneutral probability. Combining these two equations, we have

$$
p^{*} R_{u}=H \quad \text { and } \quad\left(1-p^{*}\right) R_{d}=1-H .
$$

We can now find the covariance $\operatorname{cov}^{(h)}(M R, \log R)$ from the perspective of an arbitrary investor $h \in(0,1)$. We have

$$
\begin{aligned}
\operatorname{cov}^{(h)}(M R, \log R) & =\mathbb{E}^{*}(R \log R)-\mathbb{E}^{(h)} \log R \\
& =p^{*} R_{u} \log R_{u}+\left(1-p^{*}\right) R_{d} \log R_{d}-h \log R_{u}-(1-h) \log R_{d} \\
& =(H-h) \log \frac{R_{u}}{R_{d}},
\end{aligned}
$$

where we use (B2) in the third line. The mNCC therefore holds when $h \geq H$, that is, for the representative investor and for all more optimistic investors.

EXAMPLE B3 (Heterogeneous preferences and beliefs): Consider a collection of investors who maximize next-period utility. Investor $i$ allocates a fraction $\theta_{i}$ of wealth to the risky asset, and $1-\theta_{i}$ to the riskless asset, so $\mathbb{E}^{(i)} W_{i, t+1}^{1-\gamma_{i}} /\left(1-\gamma_{i}\right)$, where $W_{i, t+1}=W_{i, t} R_{f}+\theta_{i} W_{i, t}\left(R-R_{f}\right)$. Risk aversion $\gamma_{i} \geq 1$ may be heterogeneous across investors. Beliefs are also heterogeneous: we suppose that every investor $i$ perceives the return on the market as $\operatorname{lognormal}, \log R \sim N\left(\mu_{i}, \sigma_{i}^{2}\right)$, and that $\mu_{i}-r_{f}+\frac{1}{2} \sigma_{i}^{2}=\gamma_{i} \sigma_{i}^{2}$, where $r_{f}=\log R_{f}$ is the $\log$ riskless rate. This last assumption implies (together with the first-order condition for optimal $\theta_{i}$ ) that every investor will set $\theta_{i}=1$, which clears the market. Every investor is therefore representative, and as $\gamma_{i} \geq 1$, the mNCC holds for them all.

\section{Appendix C: Additional Figures}

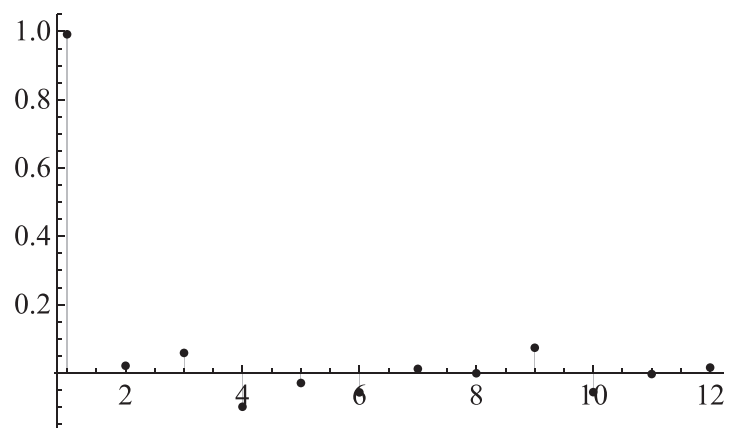

Figure C.1. Partial autocorrelations of $\boldsymbol{y}_{\boldsymbol{t}}$. Annual data, 1947 to 2017, cash-reinvestment method. 


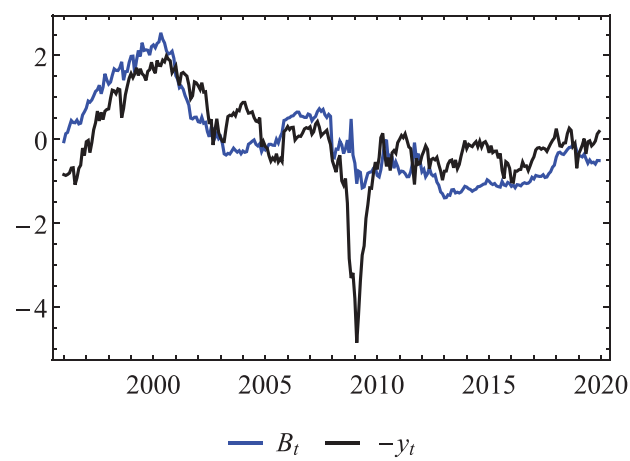

Panel A. $-y_{t}$ and $B_{t}$, standardized

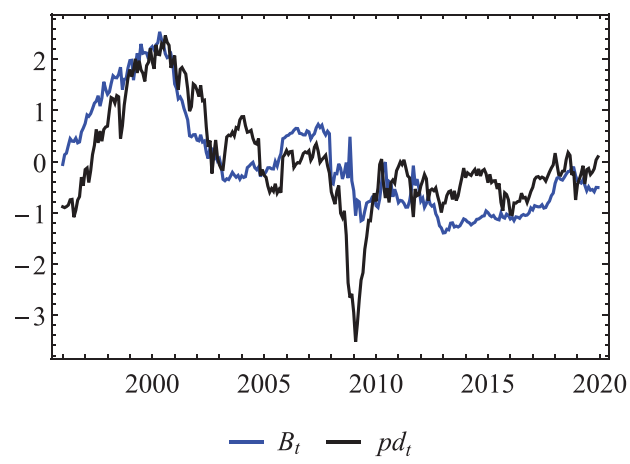

Panel B. $p d_{t}$ and $B_{t}$, standardized

Figure C.2. Standardized series of $-y_{t}, p d_{t}$, and $B_{t}$ (with $B_{t}$ calculated using predictive coefficients from the full-sample predictive regression). (Color figure can be viewed at wileyonlinelibrary.com)

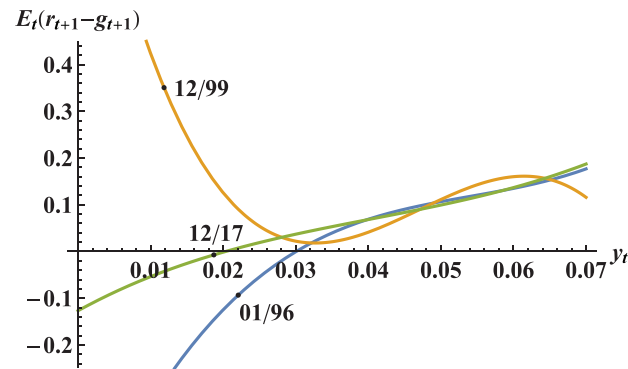

Panel A. Cubic

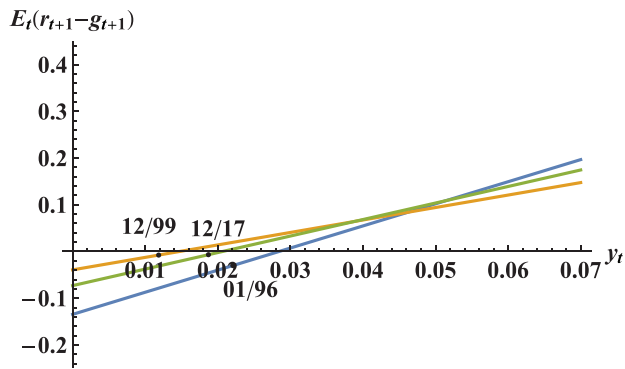

Panel B. Linear

Figure C.3. Forecasting with cubic and linear specifications at the beginning (01/96) and end (12/17) of our sample, and around the market highs in 12/99. Lines indicate the estimated functional relationship between $\mathbb{E}_{t}\left(r_{t+1}-g_{t+1}\right)$ and $y_{t}$, and dots indicate the specific values of $y_{t}$ and $\mathbb{E}_{t}\left(r_{t+1}-g_{t+1}\right)$ that happened to prevail on the relevant dates. (Color figure can be viewed at wileyonlinelibrary.com) 


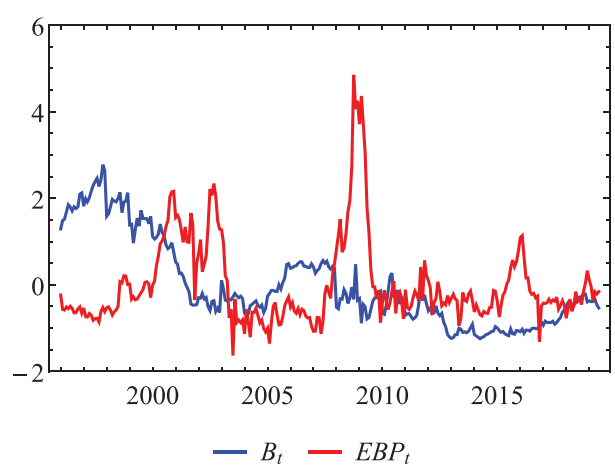

Panel A. $B_{t}$ and EBP

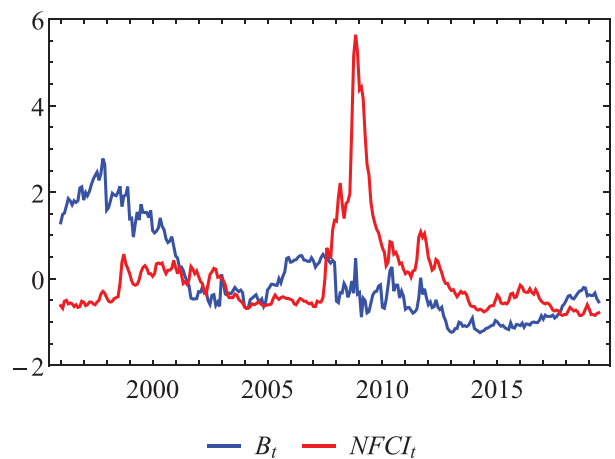

Panel C. $B_{t}$ and NFCI

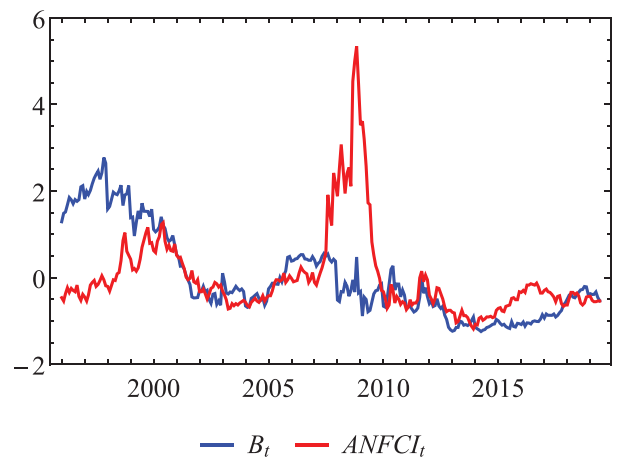

Panel E. $B_{t}$ and ANFCI

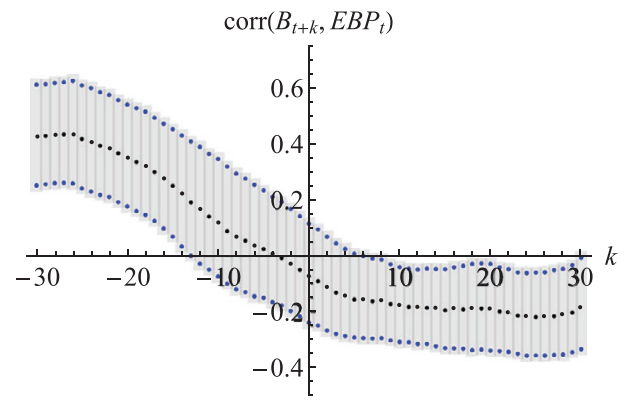

Panel B. $\operatorname{corr}\left(B_{t+k}, \mathrm{EBP}_{t}\right)$

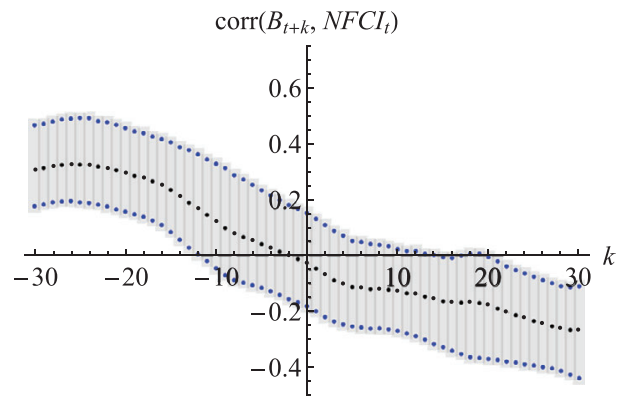

Panel D. $\operatorname{corr}\left(B_{t+k}, \mathrm{NFCI}_{t}\right)$

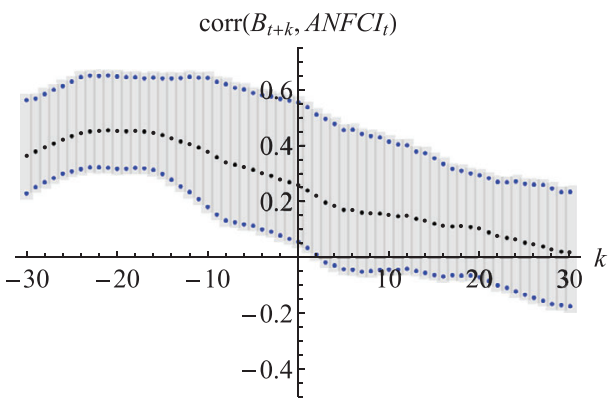

Panel F. $\operatorname{corr}\left(B_{t+k}, \mathrm{ANFCI}_{t}\right)$

Figure C.4. The relationship between $B_{t}$ and various measures of financial conditions. Shaded areas in the right panels indicate bootstrapped 95\% confidence intervals. $k$ is measured in months. (Color figure can be viewed at wileyonlinelibrary.com) 


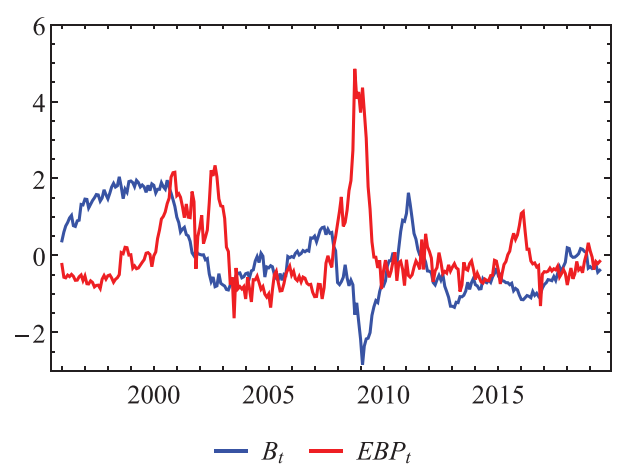

Panel A. $B_{t}$ and $\operatorname{EBP}(\mathrm{AR}(3)$, full sample)

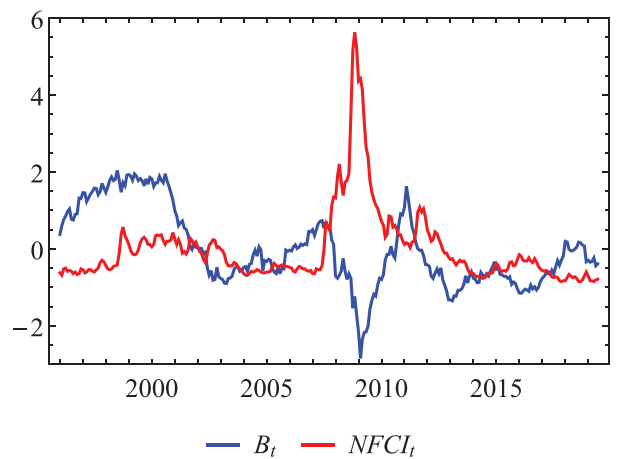

Panel C. $B_{t}(\mathrm{AR}(3)$, full sample) and NFCI

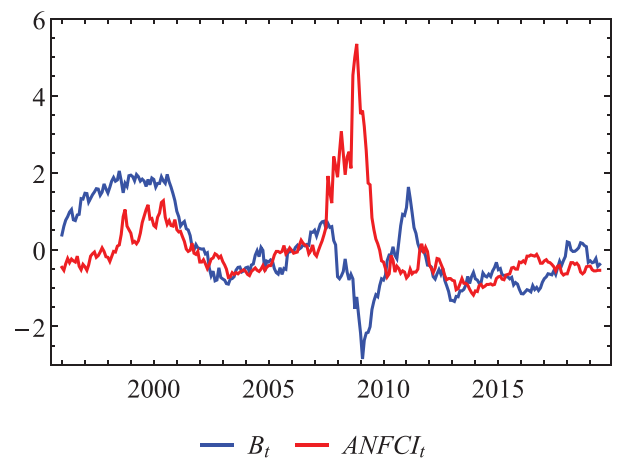

Panel E. $B_{t}(\mathrm{AR}(3)$, full sample) and ANFCI

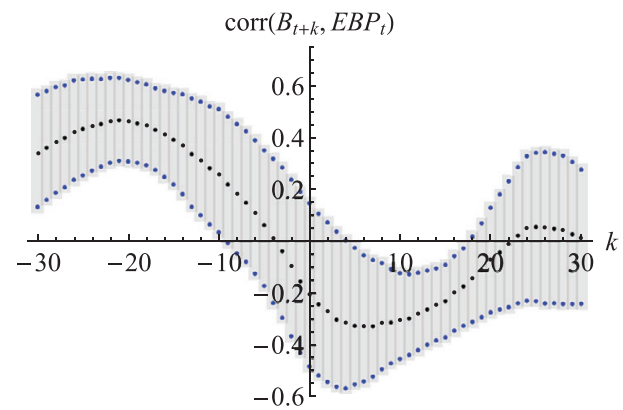

Panel B. $\operatorname{corr}\left(B_{t+k}, \mathrm{EBP}_{t}\right) \quad(\mathrm{AR}(3)$, full sample)

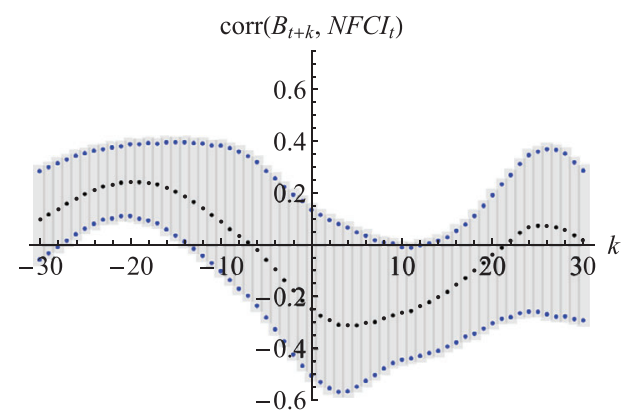

Panel D. $\operatorname{corr}\left(B_{t+k}, \mathrm{NFCI}_{t}\right)(\mathrm{AR}(3)$, full sample)

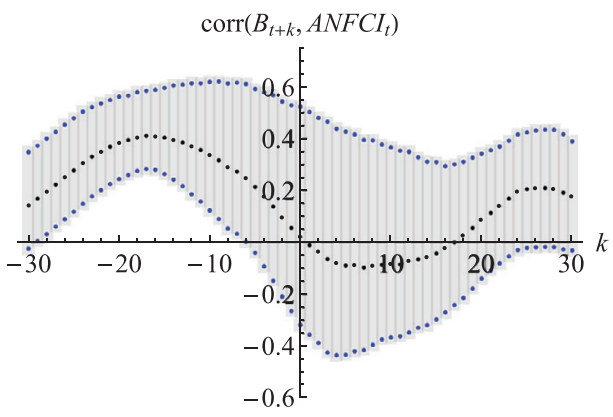

Panel F. $\operatorname{corr}\left(B_{t+k}, \mathrm{ANFCI}_{t}\right)(\mathrm{AR}(3)$, full sample)

Figure C.5. The relationship between $B_{t}$ (AR(3), full sample) and various measures of financial conditions. Shaded areas in the right panels indicate bootstrapped 95\% confidence intervals. $k$ is measured in months. (Color figure can be viewed at wileyonlinelibrary.com) 


\section{REFERENCES}

Bansal, Ravi, Dana Kiku, Ivan Shaliastovich, and Amir Yaron, 2014, Volatility, the macroeconomy, and asset prices, Journal of Finance 69, 2471-2511.

Bansal, Ravi, and Amir Yaron, 2004, Risks for the long run: A potential resolution of asset pricing puzzles, Journal of Finance 59, 1481-1509.

Barberis, Nicholas, Robin Greenwood, Lawrence Jin, and Andrei Shleifer, 2018, Extrapolation and bubbles, Journal of Financial Economics 129, 203-227.

Ben-David, Itzhak, John R. Graham, and Campbell R. Harvey, 2013, Managerial miscalibration, Quarterly Journal of Economics 128, 1547-1584.

Breeden, Douglas T., and Robert H. Litzenberger, 1978, Prices of state-contingent claims implicit in option prices, Journal of Business 51, 621-651.

Campbell, John Y., 2008, Viewpoint: Estimating the equity premium, Canadian Journal of Economics 41, 1-21.

Campbell, John Y., 2018, Financial Decisions and Markets: A Course in Asset Pricing (Princeton University Press, Princeton, NJ).

Campbell, John Y., and John H. Cochrane, 1999, By force of habit: A consumption-based explanation of aggregate stock market behavior, Journal of Political Economy 107, 205-251.

Campbell, John Y., Stefano Giglio, Christopher Polk, and Robert Turley, 2018, An intertemporal CAPM with stochastic volatility, Journal of Financial Economics 128, 207-233.

Campbell, John Y., and Robert J. Shiller, 1988, The dividend-price ratio and expectations of future dividends and discount factors, Review of Financial Studies 1, 195-228.

Carr, Peter, and Dilip Madan, 2001, Towards a theory of volatility trading, in Elyès Jouini, Jakša Cvitanić, and Marek Musiela, eds.: Option Pricing, Interest Rates and Risk Management, Handbooks in Mathematical Finance (Cambridge University Press, Cambridge).

Carr, Peter, and Liuren Wu, 2009, Variance risk premiums, Review of Financial Studies 22, 13111341.

Cochrane, John H., 2003, Stocks as money: Convenience yield and the tech-stock bubble, in William C. Hunter, George G. Kaufman, and Michael Pomerleano, eds.: Asset Price Bubbles (MIT Press, Cambridge).

De la O, Ricardo, and Sean Myers, 2021, Subjective cash flow and discount rate expectations, Journal of Finance 76, 1339-1387.

Duffie, Darrell, Nicolae Gârleanu, and Lasse H. Pedersen, 2002, Securities lending, shorting, and pricing, Journal of Financial Economics 66, 307-339.

Epstein, Larry G., and Stanley E. Zin, 1989, Substitution, risk aversion, and the temporal behavior of consumption and asset returns: A theoretical framework, Econometrica 57, 937-969.

Esary, James D., Frank Proschan, and David W. Walkup, 1967, Association of random variables, with applications, Annals of Mathematical Statistics 38, 1466-1474.

Fama, Eugene F., and Kenneth R. French, 1988, Dividend yields and expected stock returns, Journal of Financial Economics 22, 3-25.

Gilchrist, Simon, and Egon Zakrajšek, 2012, Credit spreads and business cycle fluctuations, American Economic Review 102, 1692-1720.

Goyal, Amit, and Ivo Welch, 2008, A comprehensive look at the empirical performance of equity premium prediction, Review of Financial Studies 21, 1455-1508.

Greenwood, Robin, Andrei Shleifer, and Yang You, 2019, Bubbles for Fama, Journal of Financial Economics 131, 20-43.

Hansen, Lars P., and Robert J. Hodrick, 1980, Forward exchange rates as optimal predictors of future spot rates: An econometric analysis, Journal of Political Economy 88, 829-853.

Hansen, Lars Peter, and Ravi Jagannathan, 1991, Implications of security market data for models of dynamic economies, Journal of Political Economy 99, 225-262.

Harrison, J. Michael, and David M. Kreps, 1978, Speculative investor behavior in a stock market with heterogeneous expectations, Quarterly Journal of Economics 92, 323-336.

Hong, Harrison, José Scheinkman, and Wei Xiong, 2006, Asset float and speculative bubbles, Journal of Finance 61, 1073-1117. 
Kadan, Ohad, and Xiaoxiao Tang, 2019, A bound on expected stock returns, Review of Financial Studies 33, 1565-1617.

Keim, Donald B., and Robert F. Stambaugh, 1986, Predicting returns in the stock and bond markets, Journal of Financial Economics 17, 357-390.

Kremens, Lukas, and Ian W. R. Martin, 2019, The quanto theory of exchange rates, American Economic Review 109, 810-843.

Lamont, Owen A., and Richard H. Thaler, 2003, Can the market add and subtract? Mispricing in tech stock carve-outs, Journal of Political Economy 111, 227-268.

Lintner, John, 1956, Distribution of incomes of corporations among dividends, retained earnings, and taxes, American Economic Review Papers \& Proceedings 46, 97-113.

Longstaff, Francis A., and Jiang Wang, 2012, Asset pricing and the credit market, Review of Financial Studies 25, 3169-3215.

Martin, Ian W. R., 2013, Consumption-based asset pricing with higher cumulants, Review of Economic Studies 80, 745-773.

Martin, Ian W. R., 2017, What is the expected return on the market? Quarterly Journal of Economics $132,367-433$.

Martin, Ian W. R., and Dimitris Papadimitriou, 2021, Sentiment and speculation in a market with heterogeneous beliefs, Working paper, London School of Economics.

Martin, Ian W. R., and Christian Wagner, 2019, What is the expected return on a stock? Journal of Finance 74, 1887-1929.

Nagel, Stefan, and Zhengyang Xu, 2019, Asset pricing with fading memory, Working paper, University of Chicago.

Neuberger, Anthony, 2012, Realized skewness, Review of Financial Studies 25, 3423-3455.

Ofek, Eli, and Matthew Richardson, 2003, Dotcom mania: The rise and fall of internet stock prices, Journal of Finance 58, 1113-1137.

Pástor, Ľuboš, and Pietro Veronesi, 2003, Stock valuation and learning about profitability, Journal of Finance 58, 1749-1789.

Pástor, Ľuboš, and Pietro Veronesi, 2006, Was there a Nasdaq bubble in the late 1990s? Journal of Financial Economics 81, 61-100.

Piketty, Thomas, 2014, Capital in the Twenty-First Century (Harvard University Press, Cambridge, MA).

Piketty, Thomas, 2015, About Capital in the Twenty-First Century, American Economic Review: Papers \& Proceedings 105, 48-53.

Pitt, Loren D., 1982, Positively correlated normal variables are associated, Annals of Probability 10, 496-499.

Pohl, Walter, Karl Schmedders, and Ole Wilms, 2018, Higher order effects in asset pricing models with long-run risks, Journal of Finance 73, 1061-1111.

Reinhart, Carmen M., and Kenneth S. Rogoff, 2009, This Time is Different: Eight Centuries of Financial Folly (Princeton University Press, Princeton, NJ).

Scheinkman, José A., and Wei Xiong, 2003, Overconfidence and speculative bubbles, Journal of Political Economy 111, 1183-1219.

Schneider, Paul, and Fabio Trojani, 2019, (Almost) model-free recovery, Journal of Finance 74, 323-370.

Stambaugh, Robert F., 1999, Predictive regressions, Journal of Financial Economics 54, 375-421.

\section{Supporting Information}

Additional Supporting Information may be found in the online version of this article at the publisher's website:

\section{Appendix S1: Internet Appendix. Replication Code.}

